\title{
Selective etching of injection molded zirconia-toughened alumina: towards osseointegrated and antibacterial ceramic implants
}

Quentin Flamant ${ }^{a, b, *}$, Carlos Caravaca ${ }^{c}$, Sylvain Meille ${ }^{c}$, Laurent Gremillard ${ }^{c}$, Jérôme Chevalier $^{c}$, Katia Biotteau-Deheuvels ${ }^{d}$, Meinhard Kuntz ${ }^{d}$, Rona Chandrawati ${ }^{e, 1}$, Inge K. Herrmann $^{e, 2}$, Christopher D. Spicer ${ }^{e}$, Molly M. Stevens ${ }^{e}$ and Marc Anglada, ${ }^{a, *}$

${ }^{a}$ Department of Materials Science and Metallurgical Engineering, Universitat Politècnica de Catalunya, Av. Diagonal 647, 08028 Barcelona, Spain.

${ }^{\mathrm{b}}$ Center for Research in Nano-Engineering, CRNE, Universitat Politècnica de Catalunya, C. Pascual i Vila, 15, 08028 Barcelona, Spain.

${ }^{c}$ Université de Lyon, INSA-Lyon, MATEIS UMR5510, 7 avenue J. Capelle, 69621, Villeurbanne Cedex, France.

${ }^{\mathrm{d}}$ CeramTec GmbH, 73207 Plochingen, CeramTec-Platz 1-9, Germany

e Department of Materials, Department of Bioengineering and Institute of Biomedical Engineering, Imperial College London, London SW7 2AZ, United Kingdom.

Present addresses:

${ }^{1}$ Rona Chandrawati: School of Chemical and Biomolecular Engineering, The University of Sydney, Sydney, NSW 2006, Australia

${ }^{2}$ Inge K. Herrmann: Department Materials Meet Life, Swiss Federal Laboratories for Materials Science and Technology (Empa), 9014 St Gallen, Switzerland

* Corresponding Authors

E-mail: quentin.flamant@upc.edu

E-mail: marc.j.anglada@upc.edu

Phone number: +34 934016701

Fax number: +34 934016706

Keywords: Surface modification, Roughness, Topography, Bioceramic, Drug delivery 


\section{Abstract}

Due to their outstanding mechanical properties and excellent biocompatibility, zirconiatoughened alumina (ZTA) ceramics have become the gold standard in orthopedics for the fabrication of ceramic bearing components over the last decade. However, ZTA is bioinert, which hampers its implantation in direct contact with bone. Furthermore, periprosthetic joint infections are now the leading cause of failure for joint arthroplasty prostheses. To address both issues, an improved surface design is required: a controlled micro- and nano-roughness can promote osseointegration and limit bacterial adhesion whereas surface porosity allows loading and delivery of antibacterial compounds. In this work, we developed an integrated strategy aiming to provide both osseointegrative and antibacterial properties to ZTA surfaces. The microtopography was controlled by injection molding. Meanwhile a novel process involving the selective dissolution of zirconia (selective etching) was used to produce nano-roughness and interconnected nanoporosity. Potential utilization of the porosity for loading and delivery of antibiotic molecules was demonstrated, and the impact of selective etching on mechanical properties and hydrothermal stability was shown to be limited. The combination of injection molding and selective etching thus appears promising for fabricating a new generation of ZTA components implantable in direct contact with bone. 


\section{Introduction}

Zirconia-toughened alumina (ZTA) ceramics combine the advantageous properties of monolithic alumina and zirconia: they exhibit high strength, high toughness, outstanding wear resistance and excellent biocompatibility [1-4]. Thanks to these remarkable properties, in the last decade they have become the new gold standard in orthopedics for the fabrication of ceramic bearing components. In particular, in the case of hip replacements, their superior mechanical properties when compared to alumina improve the reliability and enable the manufacture of larger femoral heads and thinner liners, providing a larger range of motion in the joint $[5,6]$.

Nevertheless ZTA is a bioinert material, which means that the host tissue forms a nonadherent fibrous capsule around the implant [7]. In the absence of adequate surface modification, this can lead to poor osseointegration and subsequent aseptic loosening [8]. For this reason, in current hip replacement systems, a metal shell with an osseointegrative surface needs to be placed between the acetabular bone and the external surface of the ceramic liner, which restricts the maximal head diameter because of the limited anatomical space [9]. This limitation confines the range of motion of the patient for maximal positions and can cause impingement, which can be followed by subluxation or even luxation [9]. It would thus be beneficial to develop surface modification processes that enable the implantation of ZTA monoblock components in direct contact with bone.

Despite the high success rate of joint replacement surgeries, approximately $10 \%$ of implants still fail within the first 10-20 years [10]. Infections are responsible for approximately $20 \%$ of these failures and have become the leading cause for arthroplasty revision [11-13]. Indeed, it is well known that biomedical implants provide a substrate for the adhesion of bacteria, which can 
proliferate and form biofilms, dramatically increasing the resistance to therapeutic agents [14]. The so-called "race for the surface" between bacteria and host cells makes it therefore critical to eliminate or contain pathogens as early as possible $[15,16]$ and there is a strong interest in developing surfaces that can prevent infection.

Osseointegration and infection prophylaxis are often treated as separated issues. However, as has been recently highlighted by Raphel et al., they are intimately related and should be addressed simultaneously [13]. The key to achieve both objectives is an adequate surface design. On the one hand, controlling topography is crucial to obtain successful osseointegration. In particular it has been shown that rough surfaces exhibit a better bone response than smooth ones and that the combination of micro- and nano-scale roughness can have synergistic effects [1719]. On the other hand, numerous surface engineering strategies have been explored to prevent infection. Most of them involve coatings, either to prevent bacterial adhesion or to release antibacterial agents [13].

There is little literature regarding surface modifications of ZTA ceramics, and, as discussed above, it would be highly valuable to develop processes that allow the design of implants with controlled micro- and nano-topography and antibacterial properties. Among the diverse surface micro-structuring techniques existing for ceramics, injection molding appears very promising [20]. In contrast to grinding or sandblasting for instance, it does not induce additional surface defects. Besides, it provides a high flexibility since it is theoretically possible to obtain any kind of micro-topography. Finally, it enables the mass production of complex components, which is an advantage from an industrial point of view. In the last decade, injection molding of ZTA has been successfully implemented by several authors [21-24]. However it has not yet been applied to surface micro-structuring which shows the need for further development. On the other hand, 
among the numerous types of coating proposed for implants, alumina with pores in the $10 \mathrm{~nm}-$ $200 \mathrm{~nm}$ range (nanoporous alumina) appears an appealing solution for the combination of osseointegrative and antibacterial properties: it can be used as a carrier for drug delivery [25-28] and in vitro studies have suggested that thanks to its nano-structure it could improve osteoblast adhesion and proliferation, increase matrix production and induce osteogenic differentiation [2931]. Nevertheless, coatings present several disadvantages; in particular they induce residual stresses and risks of delamination, which may lead to implant failure.

Long-term reliability is a major concern for orthopedic implants, and ceramics can be sensitive to surface alterations. In particular surface defects have a strong influence on their strength [32]. Furthermore, even a moderate porosity can have a substantial impact on their elastic modulus, strength and resistance to contact damage [33-35]. Finally, zirconia-containing ceramics require special attention: the tetragonal to monoclinic phase transformation, which accounts for their exceptional toughness, can occur spontaneously at low temperature in the presence of water, potentially deteriorating the material properties [36,37]. The kinetics of this phenomenon, known as low temperature degradation (LTD) or ageing, are highly sensitive to processing changes, as attested by the failure of Prozyr® zirconia femoral heads in 2002 [38]. Even if ZTA is much more resistant to LTD than monolithic zirconia, it has been shown that it can still present a certain degree of surface tetragonal to monoclinic phase transformation in the presence of water [39-41]. All these elements lead to the following conclusion: to ensure longterm reliability and patient safety, any change in the processing of zirconia-containing ceramics should be accompanied with a careful assessment of its impact on mechanical properties and ageing sensitivity, especially in the presence of porosity. 
To address the issues mentioned above, here we develop new methods for the fabrication of reliable ZTA ceramics with surface properties tailored for promoting osseointegration and preventing infections, with the perspective of enabling the implantation of ZTA components in direct contact with bone in the future. To achieve this goal, we hypothesize that surface topography should be controlled at both the micro- and the nano-scale to induce an adequate bone response, and that local drug delivery can reduce dramatically the risk of infections. Our approach was the following: samples with a tailored micro-topography were obtained by injection molding and a novel process, based on the selective dissolution of the zirconia phase (selective etching), allowed the induction of nano-roughness and the formation of an interconnected porous alumina layer. A careful assessment of the impact of selective etching on mechanical properties and ageing sensitivity was conducted and a proof of concept that the porous layer can be used as a carrier for drug delivery was demonstrated.

\section{Materials and methods}

\subsection{Fabrication of samples with a controlled micro-topography by injection molding}

The ZTA composites produced in this study were fabricated at CeramTec GmbH (Plochingen, Germany) and consisted of an alumina matrix (80 vol. \%) containing a small amount of chromia (about 0.3 wt. \%) reinforced with a secondary phase composed of yttriastabilized zirconia (Y-TZP, 17 vol. \%) and $\mathrm{SrAl}_{12} \mathrm{O}_{19}$ platelets (3 vol. \%).

An aqueous slurry containing a mixture of alumina, zirconia, strontia, chromia and yttria powders was prepared using electrosteric dispersants. Wet milling was performed in a rotary mill, using zirconia milling balls. The slurry was spray-dried without any organic additive. The ceramic powder was then mixed with an organic binder system (about 45 vol.\%), mainly 
consisting of polyethylene glycol, polyvinyl butyral and stearic acid (proprietary, confidential composition). Homogenization was achieved in a shear roller plant, where the powder-binder mixture was heated up to about $100{ }^{\circ} \mathrm{C}$ and then sheared and compressed between two rotating rollers.

Green bodies in the shape of disks were obtained by high pressure injection molding $(\mathrm{P} \approx$ 1000 bar, temperature up to $150{ }^{\circ} \mathrm{C}$ ). The air contained in the feedstock was evacuated prior to injection. The injection molding process is represented in Figure 1 and is carried out as follows: the feedstock granules are poured into the barrel of the molding machine. They are gravity fed towards the screw unit and driven along the screw until the nozzle. All along the screw unit, the feedstock is heated to get a good flowability. Then, the screw is pushed forward and injects the material through the nozzle into the mold. When the mold cavity is completely filled, the machine opens the tools and the ejector pins are activated to push the green body out of the mold.

Debinding, i.e. evaporation of the polymer from the green body was achieved with extremely slow and well controlled heating up to $350{ }^{\circ} \mathrm{C}$. The green bodies were then subjected to a three-step heat treatment. The first sintering step was performed in a gas furnace at $\mathrm{T} \approx 1500$ ${ }^{\circ} \mathrm{C}$, obtaining a relative density of $96-99 \%$. Further densification and elimination of the defects was achieved by hot isostatic pressing $\left(\mathrm{T} \approx 1400{ }^{\circ} \mathrm{C}, \mathrm{P} \approx 1200\right.$ bar $)$ in argon atmosphere. To compensate the depletion of oxygen ions in the lattice related to the previous step, specimens were subjected to a final whitening step at $\mathrm{T} \approx 1200{ }^{\circ} \mathrm{C}$. Total linear shrinkage after debinding and sintering was about $18 \%$ (final disk diameter: $20 \mathrm{~mm}$, final disk thickness: $2 \mathrm{~mm}$ ). 
To achieve different micro-topographies, the surfaces of three molds were sandblasted (Sandmaster FG 2-94, Zofingen, Switzerland; blasting material: SiC; particle size: $180 \mu \mathrm{m}$; angle: $45^{\circ}$; distance: $20 \mathrm{~mm}$ ) with increasing pressures (1 bar, 2 bar and 7 bar). The resulting surfaces, ordered from smoothest to roughest (i.e., lowest to highest pressure), will be referred to as "low", "medium" and "high" in the rest of the manuscript. Additionally, a batch of samples injected in a flat mold and a batch of polished samples were produced. The back surface of all disks was ground in order to remove the "gate", which is inherent to the injection molding process.

a)
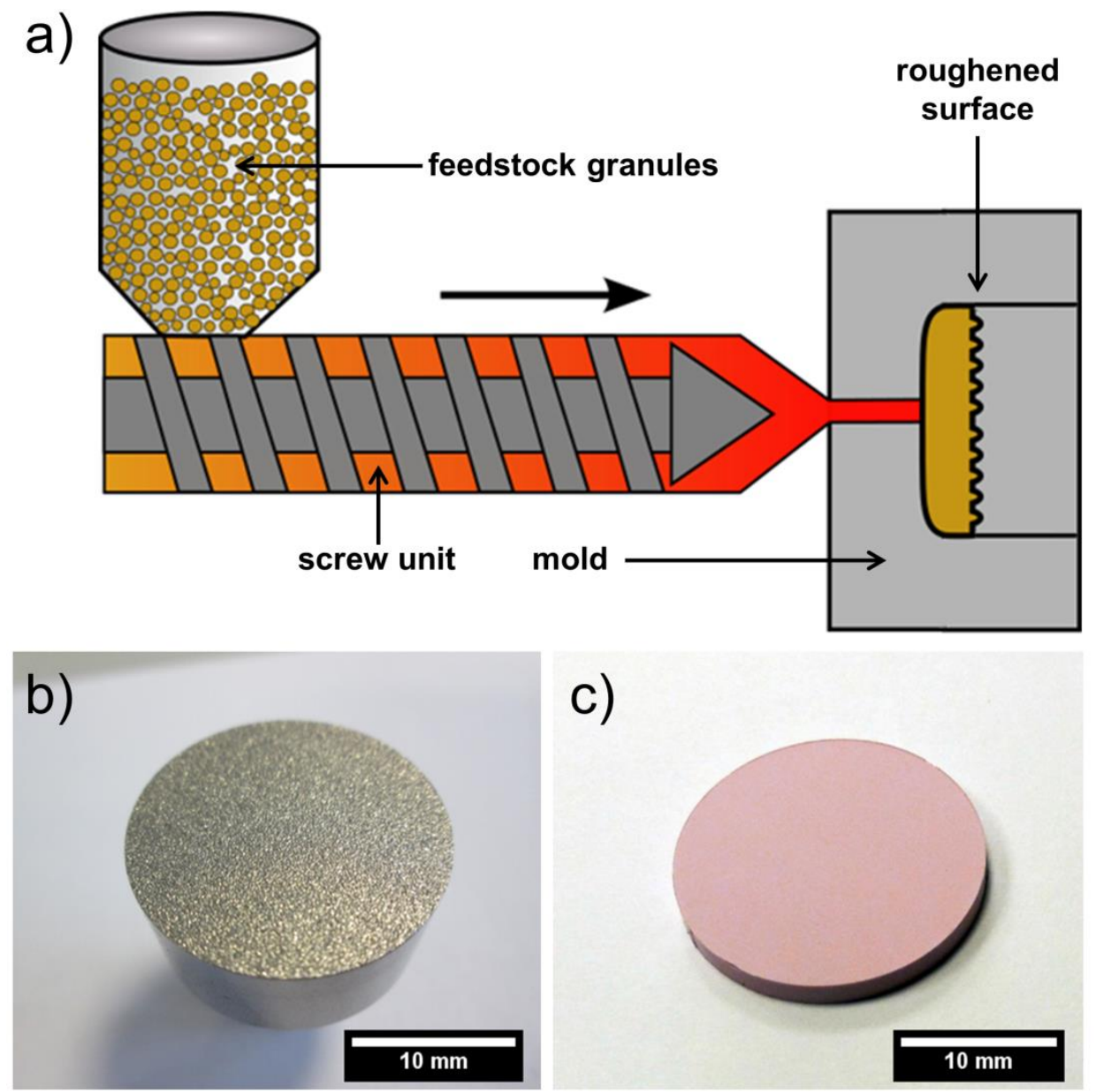

c)

Figure 1. a) Sketch of the injection molding process; b) example of mold part with a roughened surface; c) example of injected zirconia-toughened alumina disk after sintering. 


\subsection{Generation of nano-roughness and interconnected porosity by selective etching}

Specimens obtained by injection molding were successively cleaned by sonication in pure acetone, pure ethanol and deionized water in order to remove contaminants ( $5 \mathrm{~min}$ for each step). Based on the fact that zirconium dioxide can be dissolved in hydrofluoric acid (HF) [42] while $\alpha$-alumina is highly resistant to HF [43], a selective removal of the zirconia phase was achieved by immersing the samples in concentrated HF (Hydrofluoric Acid 40\% QP, Panreac, Barcelona, Spain) at room temperature for times comprised between 6 h, 1, 2, 4, 8 and 12 days. Each sample was placed in an individual high-density polyethylene flask with $4 \mathrm{~mL}$ of solution. After etching, the samples were rinsed and sonicated in deionized water. The formation of fluoride precipitates, which remained trapped into the pores, was detected when observing the surface by scanning electron microscopy (SEM, Neon40, Carl Zeiss AG, Oberkochen, Germany). To dissolve them, the specimens were immersed in $8 \mathrm{~mL}$ of concentrated $\mathrm{HCl}$ (Hydrochloric acid 37\%, Panreac, Barcelona, Spain) for $1 \mathrm{~h}$. As will be discussed later, monitoring etching time made possible to obtain either a superficial removal of zirconia, inducing nano-roughness, or an in-depth removal which resulted in the formation of both nanoroughness and interconnected porosity in a surface layer.

\subsection{Surface characterization}

\subsubsection{Surface morphology}

The surface morphology of polished and micro-rough specimens was observed by SEM after the main steps of the fabrication process (sintering, etching in HF, dissolution of the reaction precipitates in $\mathrm{HCl}$; two samples per step and per surface type). 


\subsubsection{Surface topography}

White light interferometry (WLI, Wyko 9300NT, Veeco, Oyster Bay, USA) and atomic force microscopy (AFM, Dimension 3100, Veeco, Oyster Bay, USA) in tapping mode were used to characterize micro- and nano-topography, respectively. WLI measurements were performed before selective etching on ten polished specimens and ten micro-rough specimens for each type of injection mold surface ("low", "medium", "high"; area of observation: $150 \mu \mathrm{m}$ x $150 \mu \mathrm{m}$ obtained by stitching of four images acquired at magnification 50x, resolution: 758 x 758 pixels). AFM measurements were performed on five polished samples before and after selective etching (area of observation: $50 \mu \mathrm{m} \times 50 \mu \mathrm{m}$ for roughness analysis and $10 \mu \mathrm{m} \times 10 \mu \mathrm{m}$ for imaging, resolution: 512 x 512 pixels). The roughness analysis of the data from WLI and AFM was carried out using Veeco's Vision ${ }^{\circledR}$ software. Tilt was corrected and a robust short wavelength pass Gaussian filter was applied to the data in order to separate waviness from roughness. The cut-off wavelength of the filter was set to $10 \mu \mathrm{m}$ for WLI and $1 \mu \mathrm{m}$ for AFM. In order to fully characterize the topography, one 3D roughness parameter of each of the usual categories was determined as recommended by Wennerberg et al. $[44,45]$ (Table 1). Considering the cut-off wavelengths and the lateral resolutions (micrometric for WLI, nanometric for AFM) associated to each device, the roughness measurements obtained from AFM data analysis will be referred to as "nano-roughness" whereas the measurements obtained from WLI data will be referred to as "micro-roughness".

Statistical analysis of the roughness data was performed using SPSS $®$ software (version 20, SPSS Inc., Chicago, USA). The normality of variances was verified with a Shapiro-Wilk test. The data failed to pass Levene's test of homogeneity of variances. Consequently, a one-way 
Welch's ANOVA with Games-Howell multiple comparison tests was carried out with a significance level set at $\mathrm{p}<0.05$.

Table 1. Description of the 3D roughness parameters used in this study [46-48]

\begin{tabular}{|c|c|c|c|}
\hline Symbol & Category & Name of the parameter & Description \\
\hline $\mathrm{S}_{\mathrm{a}}$ & Amplitude & Average roughness & Average of height values \\
\hline $\mathrm{S}_{\mathrm{ds}}$ & Spatial & Density of summits & $\begin{array}{c}\text { Number of summits per unit } \\
\text { area }\end{array}$ \\
\hline $\mathrm{S}_{\mathrm{dr}}$ & Hybrid & $\begin{array}{c}\text { Developed interfacial area } \\
\text { ratio }\end{array}$ & $\begin{array}{c}\text { Percentage of additional } \\
\text { surface area contributed by the } \\
\text { texture as compared to an ideal } \\
\text { plane the size of the } \\
\text { measurement region }\end{array}$ \\
\hline $\mathrm{S}_{\mathrm{ci}}$ & Functional & Core fluid retention index & $\begin{array}{c}\text { Measure, relative to } \mathrm{S}_{\mathrm{q}}(\mathrm{RMS} \\
\text { roughness), of the volume (for } \\
\text { example, of a fluid filling the } \\
\text { core surface) that the surface } \\
\text { would support from 5\% }-80 \% \\
\text { of the bearing ratio }\end{array}$ \\
\hline
\end{tabular}

\subsubsection{Surface chemistry}

To determine the influence of selective etching on the elemental composition and the chemical state of the surface, one disk was cut into three pieces, which were subjected to X-ray photoelectron spectroscopy (XPS) after respectively: cleaning, etching in HF (4 days) and immersion in $\mathrm{HCl}$. The etching time was chosen to be consistent with bacterial experiments and strength and ageing tests. The analysis was conducted using a SPECS system (Berlin, Germany) equipped with an Al anode XR50 source operating at $150 \mathrm{~W}$ and a Phoibos $150 \mathrm{MCD}-9$ detector XP. Spectra were recorded with pass energy of $25 \mathrm{eV}, 0.1 \mathrm{eV}$ steps and a pressure below $7.5 \mathrm{x}$ $10^{-9}$ mbar. Binding energies were referred to the adventitious $\mathrm{C} 1 \mathrm{~s}$ signal and background was subtracted. The identification of the local bonding environment of each element was performed 
by comparing the experimental peak positions with the data from the NIST Standard Reference Database 20, Version 4.1 (http://srdata.nist.gov/xps/).

\subsubsection{Porous layer thickness}

To monitor the evolution of the interconnected porous layer produced by selective etching over time, polished samples were cross-sectioned (two samples per time point). For short times (6 h, 1 day and 2 days), which resulted in thin layers, $10 \mu \mathrm{m}$ wide transversal sections were milled with a Focused Ion Beam (FIB, Neon40, Carl Zeiss AG, Oberkochen, Germany). Sample surfaces were protected with a thin platinum coating to flatten the surface and minimize ionbeam damage and curtain effect during milling. The final polishing of the cross-sections was performed at $500 \mathrm{pA}$. For long times (4 days, 8 days, 12 days), which resulted in thick layers, full cross-sections were obtained by cutting the entire specimens with a diamond wheel. The transversal sections were ground and polished down to a $3 \mu \mathrm{m}$ diamond suspension and subsequently observed by SEM. The mean value and standard deviation of the thickness of the layer for each individual sample were computed using the ImageJ software.

\subsection{5. $3 D$ microstructure}

To characterize the interconnected porosity induced by selective etching, a stack of 50 transversal section images (width: $15 \mu \mathrm{m}$, height: $10 \mu \mathrm{m}$, spacing: $20 \mathrm{~nm}$ ) was obtained by automatizing the FIB milling procedure described in 2.3.4. Alignment, segmentation and 3D reconstruction of the stack were performed using the Avizo® software (FEI Software, Hillsboro, Oregon) with a voxel size of $16 \mathrm{~nm}$ x $16 \mathrm{~nm}$ x $20 \mathrm{~nm}$. The porous structure was skeletonized

using Avizo® XSkeleton Pack to determine the distribution of the local radius, which is a 
measurement of the distance to the nearest boundary at every point of the skeleton. The results were used to estimate the pore size.

\subsection{Porous layer as a carrier for drug delivery}

To demonstrate that the porous layer produced by selective etching could be used as a carrier for drug delivery, micro-rough samples (with the "medium" topography) were subjected to selective etching for times comprised between 4, 8 and 12 days to achieve different porous layer thicknesses. The antibiotic gentamicin (Sigma-Aldrich, UK), commonly used in orthopedic surgery for systemic application or local delivery, was loaded either in solution or encapsulated in liposomes.

\subsubsection{Preparation of the gentamicin solutions and the gentamicin-loaded liposomes}

Solutions of the gentamicin sulfate at $10 \mathrm{mg} / \mathrm{mL}$ (stock solution), 100, 50, 25, 10, 5, 2.5 and $1 \mu \mathrm{g} / \mathrm{mL}$ were prepared by dissolution and successive dilutions in phosphate buffered saline (PBS, Sigma-Aldrich, UK). Unilamellar gentamicin-loaded liposomes were prepared by evaporation of the chloroform from a lipid solution at $50 \mathrm{mg} / \mathrm{mL}$ of DPPC (1,2-dipalmitoyl-snglycero-3-phosphocholine, phase transition temperature: $41^{\circ} \mathrm{C}$, Avanti Polar Lipids, Alabaster, USA) under nitrogen for $1 \mathrm{~h}$, followed by hydration with $10 \mathrm{mg} / \mathrm{mL}$ gentamicin solution. The solutions obtained were extruded through $100 \mathrm{~nm}$ filters (9 times) and $50 \mathrm{~nm}$ filters (11 times) to obtain liposomes with a monodisperse diameter close to the pore size, and subsequently passed through Sephadex® G-25 columns (GE Healthcare, Little Chalfont, UK) to remove excess nonloaded antibiotic. About $20 \%$ of the initial gentamicin quantity was encapsulated. Solutions were reconcentrated using Amicon® Ultra Centrifugal Filter Units (MWCO 100 kDa, Merck 
Milipore, Darmstadt, Germany), and the volume was adjusted with PBS to obtain liposomes with a lipid concentration of $1 \mathrm{mg} / \mathrm{mL}$.

\subsubsection{Loading procedure}

The loading of the gentamicin into the ZTA samples (two per etching time for each loading method) was performed in 12-well culture plates. The samples were placed in wells with $1.5 \mathrm{~mL}$ of either a gentamicin solution at $100 \mu \mathrm{g} / \mathrm{mL}$ or a gentamicin-encapsulated liposome solution at $1 \mathrm{mg} / \mathrm{mL}$ DPPC, and left overnight on a shaking plate (50 cycles/min). The samples were rinsed three times in PBS after loading.

\subsubsection{In vitro drug release experiments}

In vitro drug release experiments were carried out in duplicate in 12 -well culture plates at $37{ }^{\circ} \mathrm{C}$. Each sample loaded with gentamicin or gentamicin-encapsulated liposomes was placed into an individual well with $3 \mathrm{~mL}$ of PBS. For each time point an aliquot of $500 \mu \mathrm{L}$ was taken from the solution and replaced by $500 \mu \mathrm{L}$ of fresh PBS. Gentamicin was quantified by adapting an existing method [49-51]. An $o$-phtaldialdehyde reagent (OPA reagent) was formulated by adding $0.8 \mathrm{~g} o$-phtaldialdehyde, $20 \mathrm{~mL}$ methanol and $0.96 \mathrm{~mL}$ 2-mercaptoethanol to $180 \mathrm{~mL}$ of $40 \mathrm{mM}$ sodium borate in distilled water (all reagents from Sigma-Aldrich, UK). Aliquots of the solution to analyze were mixed in equal proportions with isopropanol and OPA reagent. Fluorescence readings were carried out in duplicate in 96-well plates with a SpectraMax M5 microplate reader (Molecular Devices, Sunnyvale, USA; excitation wavelength: $340 \mathrm{~nm}$; emission wavelength: $455 \mathrm{~nm}$ ). For each plate a calibration curve was obtained from gentamicin solutions at $100,50,25,10,5,2.5$ and $1 \mu \mathrm{g} / \mathrm{mL}$. A kinetic study of the reaction indicated that 5 min was a suitable time for the reading and that the presence of DPPC did not interfere with the 
measurement. To determine the cumulative release profiles, correction factors were applied in order to take into account the evaporation of the solution in the well and the replacement of aliquots by fresh PBS, and the total quantity released was divided by the average total sample surface area $\left(7.9 \mathrm{~cm}^{2}\right)$.

\subsubsection{Assessment of antibacterial properties against E. coli}

Table 2. Nomenclature of the samples used for the assessment of antibacterial properties

\begin{tabular}{|c|c|}
\hline Name & Surface treatment \\
\hline As sintered & None \\
\hline Etched & Selective etching (4 days) \\
\hline Etched+Loaded & Selective etching (4 days) + loading with gentamicin-encapsulated liposomes \\
\hline
\end{tabular}

Based on the in vitro release experiments, loading with gentamicin-encapsulated liposomes was selected as the best method to test antibacterial properties against $E$. coli, which is the most frequently isolated microorganism from gram-negative periprosthetic joint infections [52-54]. Three types of samples were prepared as described in Table 2 (two samples per condition). An etching time of 4 days was selected, since it was estimated that it could lead to a good compromise between the need for a thick porous layer and a limited impact on reliability. A small amount of a glycerol stock of E. coli (strain: Rosetta(DE3)pLysS, Novagen, Merck Biosciences, Nottingham, UK) was incubated in LB medium (Sigma-Aldrich, UK) at $37{ }^{\circ} \mathrm{C}$ under constant shaking overnight. The suspension was subsequently diluted to $2 \times 10^{7}$ cells $/ \mathrm{mL}$ in fresh medium. Samples were incubated in $3 \mathrm{~mL}$ of this bacterial suspension for $4 \mathrm{~h}$ at $37^{\circ} \mathrm{C}$ in 12-well culture plates. To evaluate bacteria concentration, $1 \mathrm{~mL}$ aliquots were taken from the 
supernatants for flow cytometry (Fortessa, BD Biosciences, Franklin Lakes, USA) and fixed with $2 \%$ paraformaldehyde (PFA, Sigma-Aldrich, UK) in PBS. To assess bacterial adhesion and viability on the different surfaces, staining solutions were prepared in individual wells by adding $3 \mu \mathrm{L}$ of SYTO ${ }^{\circledR} 9$ and $3 \mu \mathrm{L}$ of ethidium bromide (both from Thermo Fisher Scientific, Waltham, USA) to $2 \mathrm{~mL}$ of a $\mathrm{NaCl}$ aqueous solution at $0.85 \mathrm{wt} \%$. The samples were rinsed with the $\mathrm{NaCl}$ solution three times and then immersed in the staining solutions for 15 min at $37{ }^{\circ} \mathrm{C}$. Fluorescence imaging of the stained samples was carried out on a confocal microscope (Leica SP5, Leica Microsystems, Wetzlar, Germany) with a 20x dry objective. Measurements of surface area covered by living E. coli cells were carried out using the ImageJ software (three images per specimen).

\subsection{Contact behavior and mechanical properties of the porous layer}

\subsubsection{Experiments}

For the study of the mechanical properties and local contact behavior of the porous layer produced by selective etching, two polished samples were fabricated and one of them was selectively etched for 12 days (thickness of the porous layer: $27 \mu \mathrm{m}$ ). Instrumented nanoindentation tests were carried out with a MTS Nanoindenter XP (Eden Prairie, USA) equipped with a continuous stiffness measurement (CSM) module and a diamond spherical tip (nominal radius: $50 \mu \mathrm{m}$ ). Due to the difficulty to machine diamond at such a small scale, the real shape of the tip can differ substantially from a perfect sphere. For this reason, the real tip shape was measured by AFM. A Python script was developed and used to extract the curve $a=f\left(h_{c}\right)$ with $a$ the contact radius and $h_{c}$ the contact depth, which was fitted with a power law of the type $a=A+B \times h_{c}{ }^{C}$. The best fitting parameters were adjusted by performing calibration tests 
against reference materials with well-known elastic moduli (pyrocarbon, fused silica and tungsten, see supplementary information).

Indentations of the porous layer were performed up to a maximum load of $7.5 \mathrm{~N}$ (maximum indentation depth: $~ 3.5 \mu \mathrm{m}$ ) and under a constant deformation rate of $0.05 \mathrm{~s}^{-1}$ with an interindentation spacing of $100 \mu \mathrm{m}$ (3x3 arrays). At maximum penetration, the circular contact area was very large in comparison to the pore size (contact radius: $\sim 17 \mu \mathrm{m}$ ). The contact point was corrected implementing the method proposed by Moseson et al. [55] and the polished sample was used as a reference for stiffness correction. The models chosen to interpret analytically the indentation data were those of Hertz, Oliver and Pharr and Tabor, following the approach of He and Swain [56].

Finally, profiles of residual indents were measured with a laser scanning confocal microscope (LEXT, Olympus, Tokyo, Japan) and a FIB cross-section of an indentation was realized in order to identify the deformation mechanism of the porous layer under compression and to detect potential damage and densification.

\subsubsection{Numerical analysis}

Based on previous works on porous ceramics [57,58], an inverse finite element (FE) analysis was used to identify the properties of the porous layer. Lowest, average and highest experimental load-displacement curves were used for the identification, and the plateau separating loading and unloading sections was removed. The FE simulation was carried out using the ABAQUS/Standard software (Dassault Systèmes, Simulia, Vélizy-Villacoublay, France). The model consisted of a 2D axisymmetric mesh (available in supplementary information) which contained approximately 6000 elements (CAX8 and CAX8-R) and was 
refined towards the contact zone with an element size of about $0.5 \mu \mathrm{m}$. Loading was achieved by imposing a quasi-static vertical displacement ("hard contact", sliding formulation: finite sliding, discretization method: surface to surface). The indenter and the bulk of the sample were modeled as elastic materials, whereas the porous layer was modeled using a modified DruckerPrager/cap-plasticity criterion which accounts for hydrostatic pressure sensitivity of material failure through two surfaces: the Drucker-Prager surface for shear failure and the cap surface for high hydrostatic pressure failure [59] (see Appendix A for the definition of the model and of the related parameters). The increase in elastic modulus associated to densification of the porous material was taken into account using ABAQUS user subroutine USDFLD (time increment was kept small enough to maintain the accuracy of the solution).

The inverse identification was carried out using the MIC2M software (http://mic2m.univfcomte.fr/). The Poisson ratio $v$ of the porous layer was set to 0.22 (taken from literature for porous alumina [35]), the cap eccentricity $R$ was set to 0.25 to obtain a cap yield surface neither too circular nor too steep, $\alpha$ (a small number used to define the transition yield surface) was set to 0.01 and $W$ (the porous volume fraction) was set to 0.17 . A preliminary calculation showed that variation of the friction coefficient $f$ between the indenter and the sample surface had negligible effect on the results and $f$ was set to 0.1 . The parameters to identify were the elastic modulus of the porous layer $(E)$, the yield stress in simple compression $\left(\sigma_{c}\right)$, the angle of friction $(\beta)$, the initial hydrostatic compression yield stress $\left(p_{b 0}\right)$ and the maximum plastic volumetric strain rate $(D)$. 


\subsection{Impact of selective etching on strength and ageing kinetics}

To assess the impact of selective etching on strength and ageing sensitivity, 48 flat samples were fabricated. Half of them were polished down to a $1 \mu \mathrm{m}$ diamond suspension and annealed at $1200{ }^{\circ} \mathrm{C}$ for $10 \mathrm{~min}$ in air using heating and cooling rates of $5{ }^{\circ} \mathrm{C} / \mathrm{min}$, and they were divided into four groups as described in Table 3. The polishing was introduced to remove surface defects and the annealing to remove residual stresses. The annealing temperature was chosen based on a preliminary study involving an indentation technique [60] in which $1200{ }^{\circ} \mathrm{C}$ was found to be the minimum annealing temperature capable of removing the majority of residual stresses without affecting significantly the grain size. The etching time was chosen to be the same as for the testing of antibacterial properties (2.4.4), and the thickness of the porous layer was measured by performing cross-sections on three samples from each etched group (same procedure as described in 2.3.4).

Table 3. Nomenclature of the sample groups used for strength and ageing kinetics testing

\begin{tabular}{|c|c|}
\hline Name of the group & Surface treatment \\
\hline As sintered (AS) & None \\
\hline Polished+Annealed (P+A) & Polishing + Annealing $\left(1200{ }^{\circ} \mathrm{C}, 10\right.$ min $)$ \\
\hline As sintered+Etched (AS+E) & Selective etching $(4$ days $)$ \\
\hline $\begin{array}{c}\text { Polished+Annealed+Etched } \\
(\mathrm{P}+\mathrm{A}+\mathrm{E})\end{array}$ & $\begin{array}{c}\text { Polishing }+ \text { Annealing }\left(1200^{\circ} \mathrm{C}, 10 \text { min }\right)+\text { Selective } \\
\text { etching }(4 \text { days })\end{array}$ \\
\hline
\end{tabular}

\subsubsection{Biaxial flexural strength testing}

The biaxial flexural strength of ten samples from each group was assessed by 3-balls-on-3balls testing with a "sphere-in-line" configuration [61]. The specimens were tested in a universal 
testing machine (Model 8502, Instron Corp., Canton, USA) in air up to fracture of the specimen, using a constant test speed of $0.5 \mathrm{~mm} / \mathrm{min}$. The radius of the inner sphere location circle was $R_{1}$ $=4.08 \mathrm{~mm}$ and the ratio of outer to inner sphere circles was $\mathrm{R}_{1} / \mathrm{R}_{2}=2$. The fracture strength was calculated using a numerical approximation of the maximum tensile stress:

$$
\sigma_{\max }=f \times \frac{F}{t^{2}}
$$

where $F$ is the applied load on failure, $t$ the sample thickness and $f$ a dimensionless factor. For $R / R_{l}=2.25$ ( $R$ being the diameter of the test samples), $f$ can be calculated with the following formula [61]:

$$
f=0.656\left(\frac{t}{R_{1}}\right)^{-0.196}+0.274\left(\frac{t}{R_{1}}\right)^{-0.448} \times v
$$

where $t$ is the thickness of the sample and $v$ is the Poisson ratio. In the present study, $R / R_{l}=$ 2.45, nevertheless it is still possible to use equation (2) with an error inferior to $5 \%$ [62].

Statistical analysis of the strength testing results was performed using SPSS® software (version 20, SPSS Inc., Chicago, USA). A two-way ANOVA with a 5\% significance level was used to evaluate the effects of polishing and selective etching. The data was log-transformed prior to analysis. The normality and the homogeneity of variances were verified with respectively a Shapiro-Wilk test and a Levene test.

The variability of the strength was analyzed using the Weibull distribution function:

$$
P_{F}(\sigma)=1-\exp \left(-\left(\frac{\sigma}{\sigma_{0}}\right)^{m}\right)
$$

where $P_{F}$ is the cumulative probability of failure, $\sigma$ is the fracture strength, $\sigma_{0}$ is the Weibull characteristic strength, and $m$ is the Weibull modulus. For the evaluation of $m$ and $\sigma_{0}$ the 
measured strength data were ranked in increasing order and numbered from 1 to $N$. Then the single strength values $\sigma_{i}$ were related to the failure probability $P_{F i}$ according to the following relation:

$$
P_{F i}=\frac{i-0.5}{N}
$$

where $i$ is the ranking number and $N$ is the total number of measurements (for a more detailed description of the methodology, see for instance Munz et al. [63]). Finally, the 90\% confidence bounds for $m$ and $\sigma_{0}$ were determined according to ASTM C1239-00.

\subsubsection{Ageing kinetics}

Two samples from each group were subjected to hydrothermal degradation tests. The tests were performed in an autoclave, at $134{ }^{\circ} \mathrm{C}, 100 \%$ steam atmosphere at $0.2 \mathrm{MPa}$ pressure for times up to $600 \mathrm{~h}$. This time is far beyond the requirement of $10 \mathrm{~h}$ recommended by the ISO 6474-2 standard and according to Chevalier et al. (2009) [41] it is equivalent to about 1500 years at $37^{\circ} \mathrm{C}$. It is thus extremely conservative but monitoring the long-term evolution of the monoclinic phase content allows amplifying potential differences between groups. The specimens were analyzed by X-ray diffraction (XRD) (Model D8, Bruker AXS, Madison, USA) using $\mathrm{Cu}-\mathrm{K}_{\alpha}$ radiation to detect and quantify the tetragonal-monoclinic transformation. The monoclinic fraction was determined using the relation proposed by Toraya et al. [64]:

$$
V_{m}=1.311 \frac{I_{m}(\overline{1} 11)+I_{m}(111)}{I_{t}(101)+1.311\left[I_{m}(\overline{1} 11)+I_{m}(111)\right]}
$$

where $V_{m}$ is the monoclinic volume fraction, $I_{m}(\overline{1} 11)$ and $I_{m}(111)$ are the intensities of the monoclinic peaks and $I_{t}(101)$ is the intensity of the tetragonal peak. 


\section{Results}

\subsection{Surface characterization of injection molded and selectively etched samples}

3D topographical images obtained by WLI showed that injection molded ZTA samples presented very diverse micro-topographies (Figure 2). As can be observed on the WLI images, increasing pressure during sandblasting led to an increased roughening of the mold surface and consequently of the surface of injected samples: the "high" surface displayed the broadest and deepest valleys / highest peaks, whereas the "low" surface appeared relatively flat (but rougher than the polished surface), and the "medium" surface was an intermediary between both. Furthermore, the micro-roughness analysis showed that a large range of values could be obtained for the average roughness ("low" surface: $S_{a}=176 \pm 15 \mathrm{~nm}$; "medium" surface: $S_{a}=$ $330 \pm 28 \mathrm{~nm}$; "high" surface: $S_{a}=417 \pm 16 \mathrm{~nm}$; polished surface, for reference: $S_{a}=13 \pm 1 \mathrm{~nm}$ ) and the developed interfacial area ratio ("low" surface: $S_{d r}=45 \pm 6 \%$; "medium" surface: $S_{d r}=$ $82 \pm 12 \%$; "high" surface: $S_{d r}=99 \pm 11 \%$; polished surface, for reference: $S_{d r}=0.4 \pm 0.2 \%$ ). Statistical analysis of the roughness data evidenced that differences between groups were significant (Welch's ANOVA resulted in $\mathrm{p}<0.001$ for each roughness parameter). For both $S_{a}$ and $S_{d r}$, differences between each type of surface were strongly significant ( $\mathrm{p} \leq 0.001$ for each pairwise comparison using Games-Howell test), while for $S_{d s}$ differences were significant only when comparing the polished surface to the others ( $\mathrm{p} \leq 0.001$ in each case) and for $S_{c i}$ differences were significant only when comparing the "medium" surface to the others ( $p \leq$ 0.001 in each case). Finally, the best correlation between mold and sample roughness was obtained for the "low" surface, while the "medium" and "high" sample surfaces were slightly smoother than the "medium" and "high" mold surfaces, respectively (see supplementary information). 

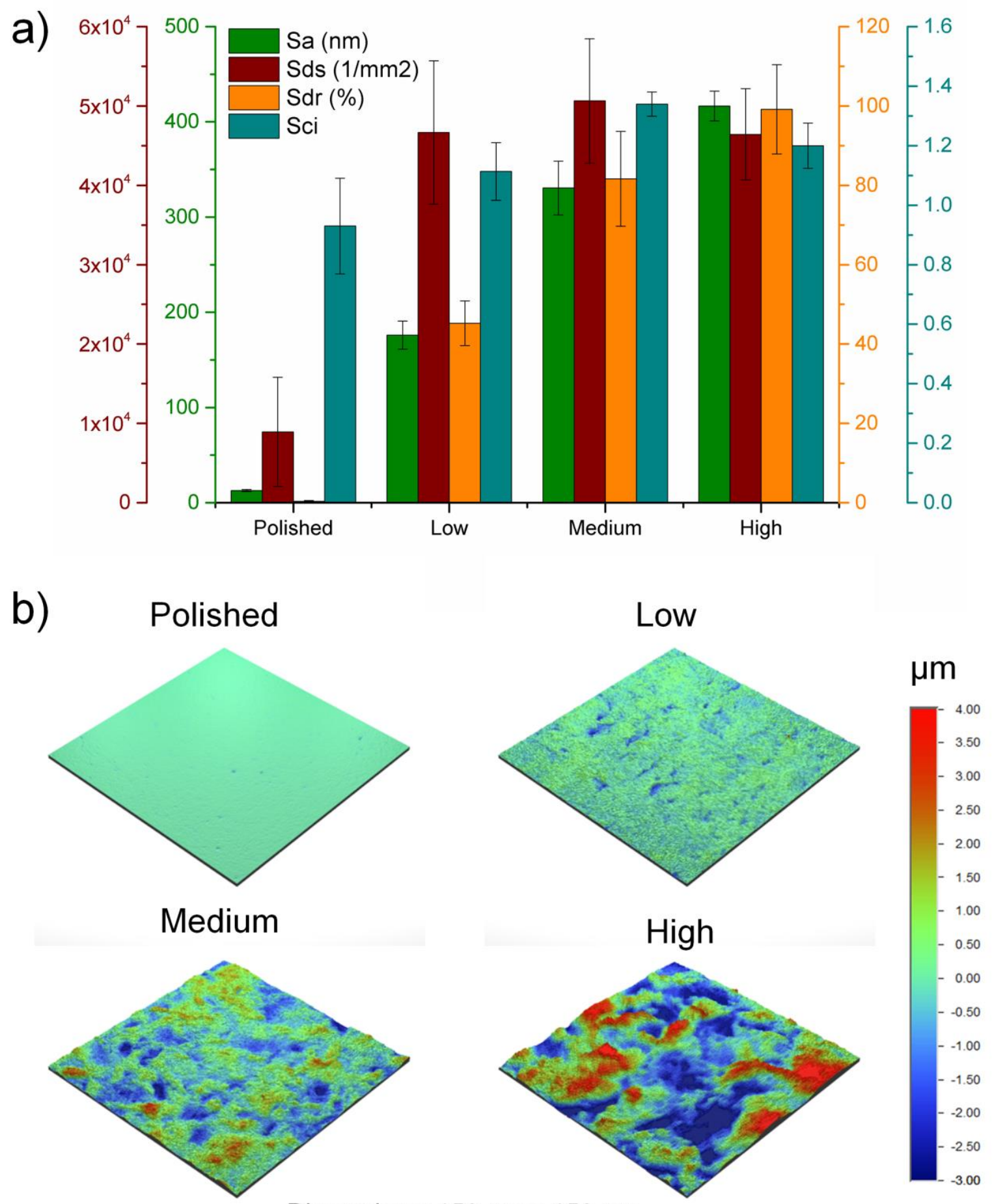

Dimensions: $150 \mu \mathrm{m} \times 150 \mu \mathrm{m}$

Figure 2. White light interferometry measurements at the surface of injection molded zirconia toughened alumina samples with different induced micro-topographies: a) roughness analysis (10 samples per group); b) 3D topographical images. "Low", "Medium" and "High" designate micro-topographies obtained from increasingly rough molds. 
Regarding selective etching, the zirconia phase was successfully removed by immersion in $\mathrm{HF}$ whereas neither the alumina matrix nor the $\mathrm{SrAl}_{12} \mathrm{O}_{19}$ platelets were affected, which allowed the integrity of the micro-topography obtained by injection molding to be preserved (Figure 3). The formation of fluoride precipitates, which remained trapped in the pores of the surface, was detected. Energy dispersive spectroscopy evidenced that they were mainly composed of yttrium and fluorine. Some of them had an octahedral shape suggesting the presence of $\mathrm{YF}_{3}$ crystals, similar to those observed when etching Y-TZP [65]. XPS analysis confirmed the presence of $\mathrm{YF}_{3}$ chemical bonds and additionally suggested the existence of zirconium oxyfluorides (Table 4). Inspection of the specimens by SEM evidenced that all precipitates were successfully removed by immersion in $\mathrm{HCl}$ (Figure 3).

The selective etching process induced a substantial increase in nano-roughness of the polished samples (Figure 4). Indeed, the average roughness $\left(S_{a}\right)$ was multiplied by 10 with respect to the polished surface (polished: $S_{a}=2.1 \pm 0.3 \mathrm{~nm}$; selectively etched: $S_{a}=21 \pm 4.6$ $\mathrm{nm}$ ) and the developed interfacial area ratio $\left(S_{d r}\right)$ was multiplied by 100 (polished: $S_{d r}=0.06 \pm$ $0.03 \%$; selectively etched: $S_{d r}=5.4 \pm 1.7 \%$ ). On the other hand, the density of summits $S_{d s}$ (polished: $S_{d s}=2.4 \times 10^{5} \pm 1.9 \times 10^{5} \mathrm{~mm}^{-2}$; selectively etched: $S_{d s}=1.2 \times 10^{5} \pm 1.1 \times 10^{5} \mathrm{~mm}^{-2}$ ) and the core fluid retention index $S_{c i}$ (polished: $S_{c i}=0.91 \pm 0.19$; selectively etched: $S_{c i}=0.39 \pm$ $0.06)$ decreased. In terms of surface chemistry, the most notable changes were an increase in the fluorine content and a decrease in the zirconium content (Figure 5).

The thickness of the layer affected by selective etching depended on etching time (Figure 6a,c). During a short time period $(t=6 \mathrm{~h})$, only the superficial zirconia grains were dissolved, leaving the bulk unaffected. For longer etching times ( $t \geq 24 \mathrm{~h}$ ), an interconnected porous layer was produced. The FIB/SEM tomography provided evidence that with the exception of some 
isolated grains the zirconia phase was percolated (Figure 6-b). The local radius distribution appeared to be centered at approximately $50 \mathrm{~nm}$ with a maximum at $160 \mathrm{~nm}$, which can be considered as the maximal pore size (Figure 6-d). It was not possible to determine the minimum of the distribution with certainty because of the limit of resolution fixed by the voxel size.

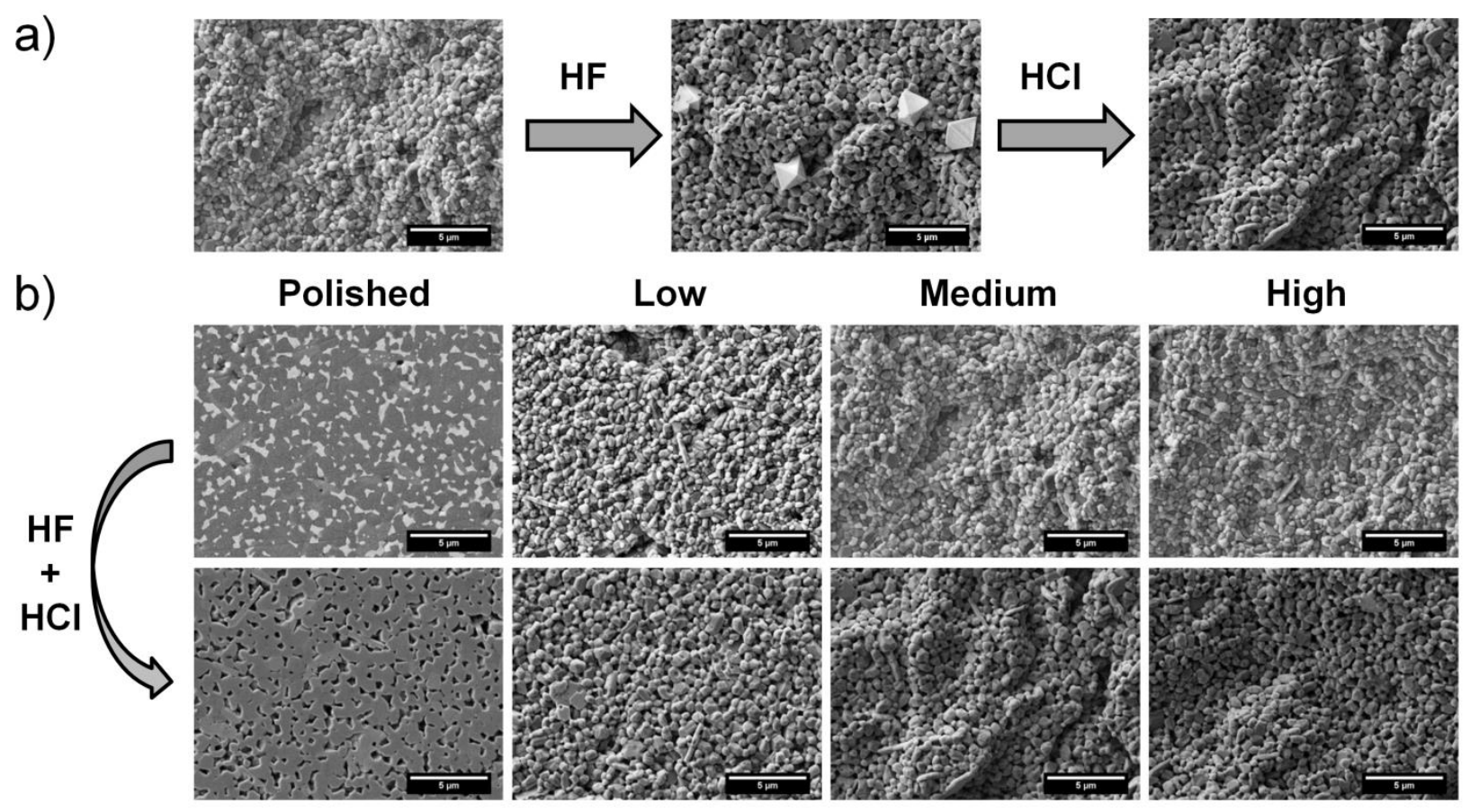

Figure 3. a) Scanning electron microscopy observations of the surface of zirconia toughened alumina at the different steps of the selective etching process, evidencing the formation of fluoride precipitates during $\mathrm{HF}$ etching and their subsequent removal in $\mathrm{HCl}$; b) scanning electron microscopy observations of the surface of injection molded samples with different micro-topographies before and after selective etching. "Low", "Medium" and "High" designate micro-topographies obtained from increasingly rough molds. Scale bars: $5 \mu \mathrm{m}$. 
a)

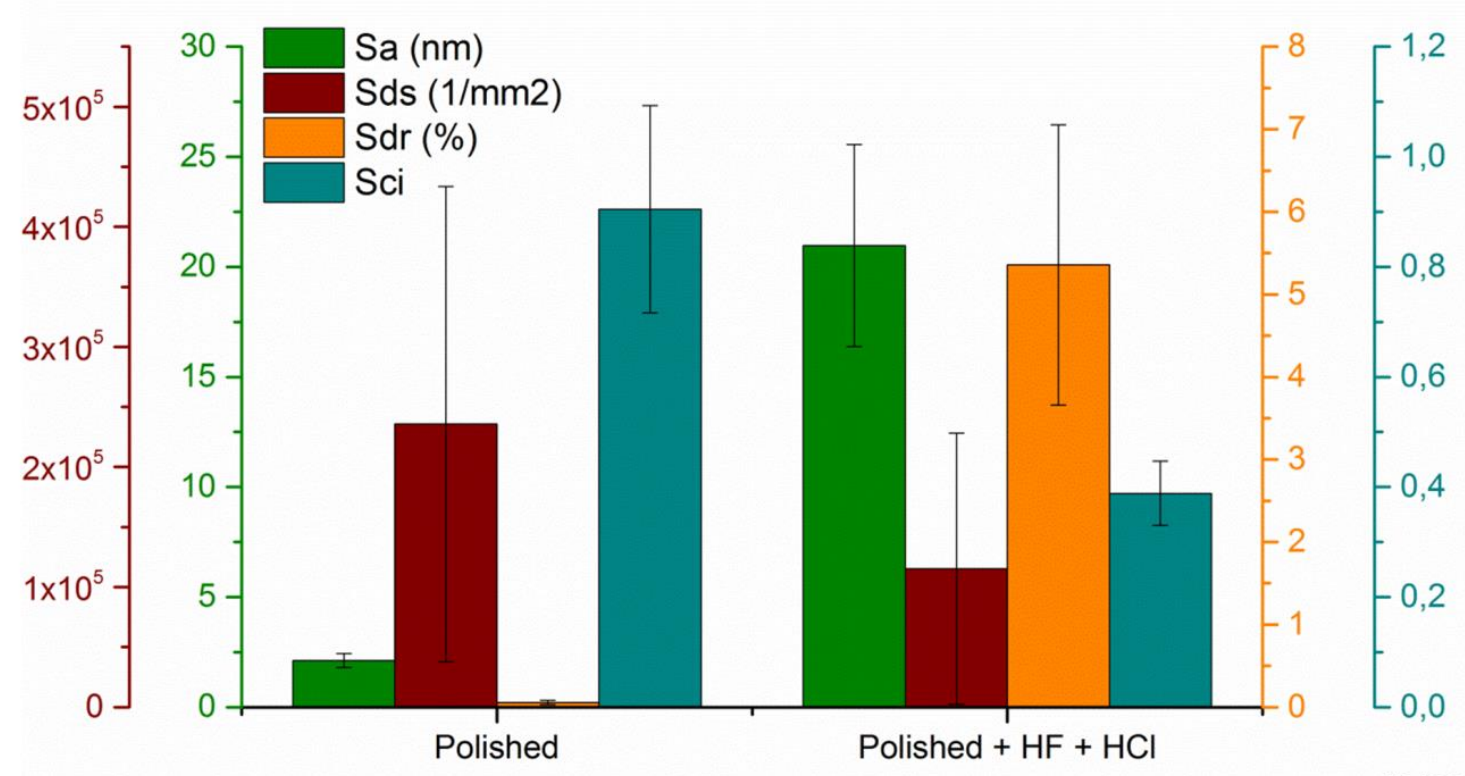

b)

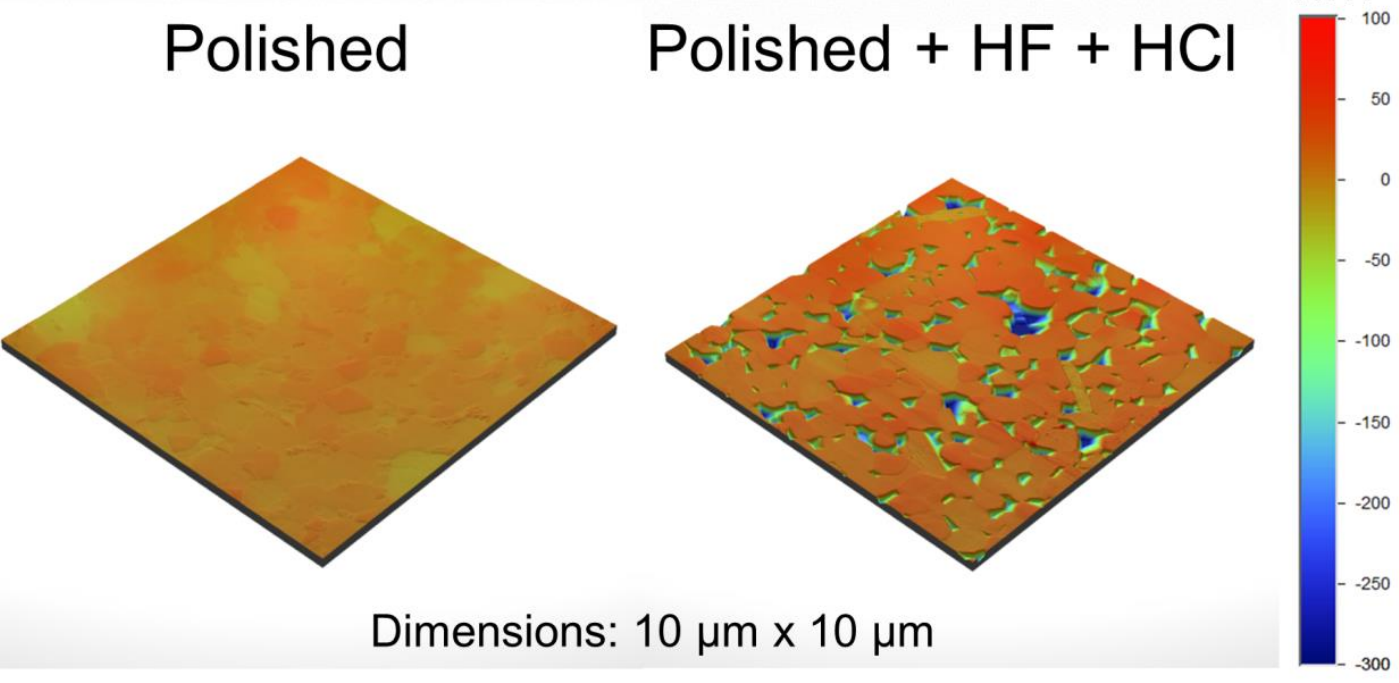

Figure 4. Atomic force microscopy measurements at the surface of polished zirconia toughened alumina samples before and after $\mathrm{HF}$ and $\mathrm{HCl}$ treatment: a) roughness analysis (5 samples per group, area of measurement: $50 \mu \mathrm{m} \times 50 \mu \mathrm{m}$ ); b) 3D topographical images (area of measurement: $10 \mu \mathrm{m} \times 10 \mu \mathrm{m})$. 

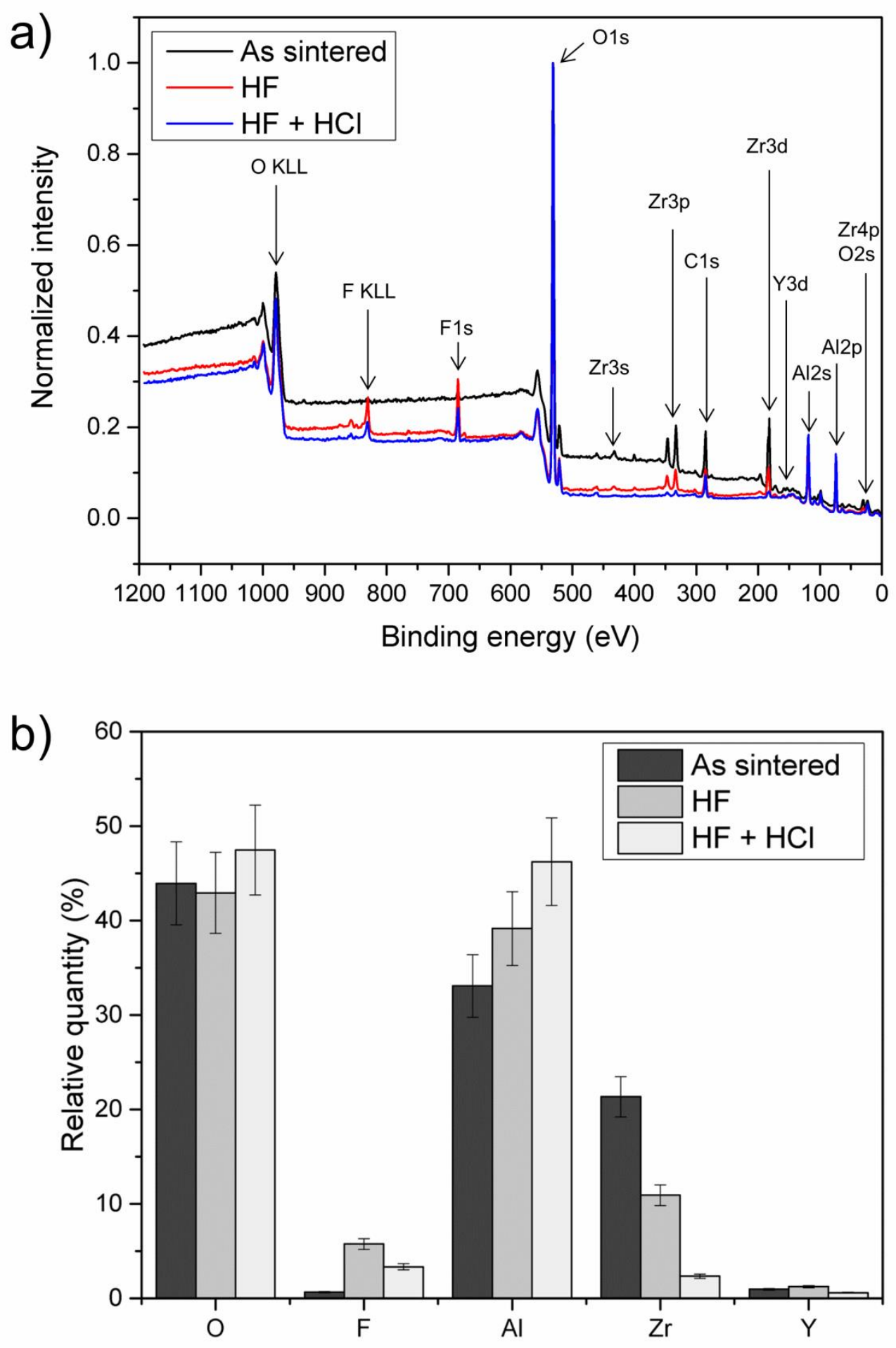

Figure 5. XPS analysis of the surfaces of an "as sintered" zirconia toughened alumina sample, an $\mathrm{HF}$ treated sample and an $\mathrm{HF}+\mathrm{HCl}$ treated sample: a) full spectra normalized to the O1s peak intensity; b) quantitative elemental analysis. Error bars represent the typical uncertainty (10\%) associated to XPS quantitative measurements. 
Table 4. Identification of the peaks of the XPS high-resolution spectra of Figure 5

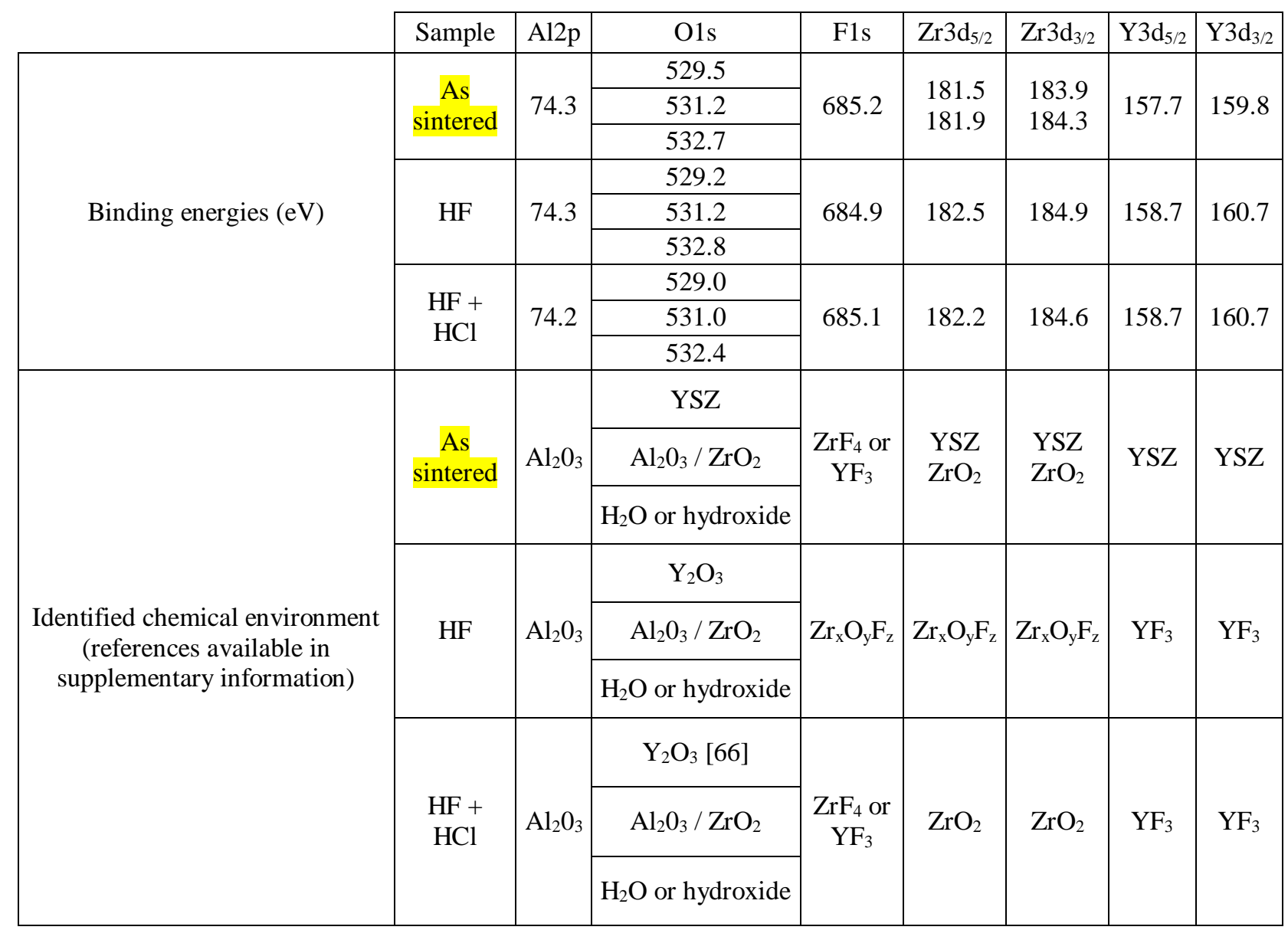

YSZ: Yttria stabilized zirconia 

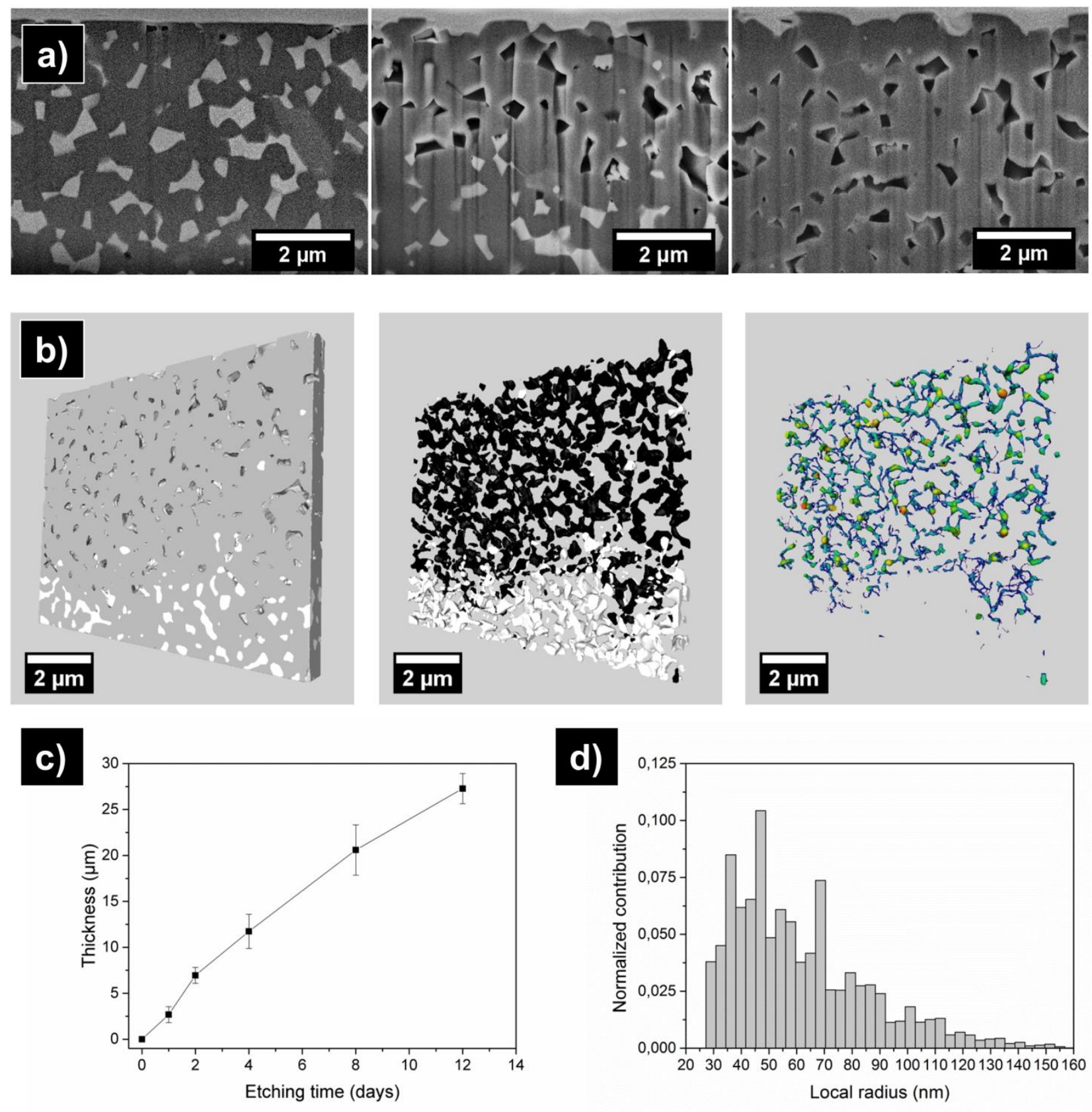

Figure 6. a) FIB cross-sections of polished zirconia toughened alumina samples after $6 \mathrm{~h}$ (left), $24 \mathrm{~h}$ (middle) and $48 \mathrm{~h}$ (right) of immersion in $\mathrm{HF}$, evidencing the progressive removal of zirconia over time (zirconia appears in white, alumina in grey and pores in black); b) FIB/SEM tomography of the surface after selective etching (4 days): external view (left), internal structure with pores in black and zirconia in white (middle), skeleton of the porous structure (right); c) evolution of the porous layer thickness over time for a polished surface. Error bars represent the combined standard deviations of single samples; d) local radius distribution of the porous structure. 


\subsection{Proof of concept: use of the porous layer as a carrier for drug delivery}

It was found that the thickness of the porous layer strongly depended on the surface state. In particular the layer was much thicker on the back surface of the disks, which was ground after sintering (see supplementary information), possibly because of combined effects of residual stresses and machining defects. Because this surface has to be taken into account for the release, each sample used for drug delivery was cross-sectioned and observed following the procedure described in 2.3.4 and the thickness value was reported as an average over the whole specimen (Figure 7).

Impregnation of the samples with gentamicin solution led to small quantities of drug loaded and released (about $16 \mu \mathrm{g} / \mathrm{sample}$ which corresponds to $2 \mu \mathrm{g} / \mathrm{cm}^{2}$ ), and there was no substantial influence of the porous layer thickness (Figure 7-a). This is probably due to the fact that alumina surface and gentamicin are both positively charged, which leads to poor adsorption. Liposome encapsulation enabled an increased amount of gentamicin loaded (up to about $80 \mu \mathrm{g}$ which corresponds to $10 \mu \mathrm{g} / \mathrm{cm}^{2}$ ), and the quantity loaded and released was roughly proportional to the porous layer thickness (Figure 7-b). This likely results from the higher affinity of the lipid to the alumina surface, as attested by quartz crystal microbalance measurements (see supplementary information), and from the greater size of the liposomes compared to the free gentamicin, which may thus remain trapped in the pores.

Bacteria cultures evidenced two effects (Figure 7-c,d). On the one hand the selectively etched surface reduced the bacterial adhesion as compared to the "as sintered" surface, even in the absence of loaded antibiotic (surface area covered by living $E$. coli after 4 h: $5 \%$ vs. $12 \%$ ). On the other hand, samples loaded with gentamicin-encapsulated liposomes limited the growth of 
bacteria in the medium and consequently reduced the surface area covered by living E. coli as compared to non-loaded specimens (bacterial concentration after $4 \mathrm{~h}: 2.8 \times 10^{7}$ cells $/ \mathrm{mL}$ vs. $1.8 \times 10^{8}$ cells $/ \mathrm{mL}$; surface area covered by living E. coli after 4 h: $0.4 \%$ vs. $5 \%$ ).

\subsection{Impact of selective etching on reliability}

\subsubsection{Contact behavior and mechanical properties of the porous layer}

Indentation hardness vs. strain analytical curves showed a plateau at $8 \mathrm{GPa}$ for the selectively etched sample and $20 \mathrm{GPa}$ for the polished sample (see supplementary information). The observation of the surface and cross-section of an indentation demonstrated that the deformation of the porous layer was quasi-plastic with a limited elastic recovery (Figure 8-a,b,c). No cracks were detected on the surface around the indent and densification could be observed below the residual imprint, which justifies the introduction of a cap in the Drucker-Prager plasticity model. The inverse FE identification carried out with the different experimental curves led to stable values for the elastic modulus $(E)$ and the yield stress in simple compression $\left(\sigma_{c}\right)$ whereas a moderate fluctuation was observed for the initial hydrostatic compression yield stress $\left(p_{b 0}\right)$, and important variations occurred for the friction angle and the maximum plastic volumetric strain rate $(D)$ (Table 5). The elastic modulus values were in good agreement with that obtained by theoretical calculation for porous alumina using Roberts and Garboczi model [35] $\left(E_{\text {theoretical }}=277 \mathrm{GPa}\right.$, considering overlapping spherical pores), but slightly superior to that computed from the analytical analysis of the indentation data $\left(E_{\text {analytical }}=215 \mathrm{GPa}\right.$, see supplementary information). Simulations with the identified parameters led to realistic results, with good consistency between experimental and numerical load-displacement curves, residual imprints and densification behavior (Figure 8-d,e,f). 

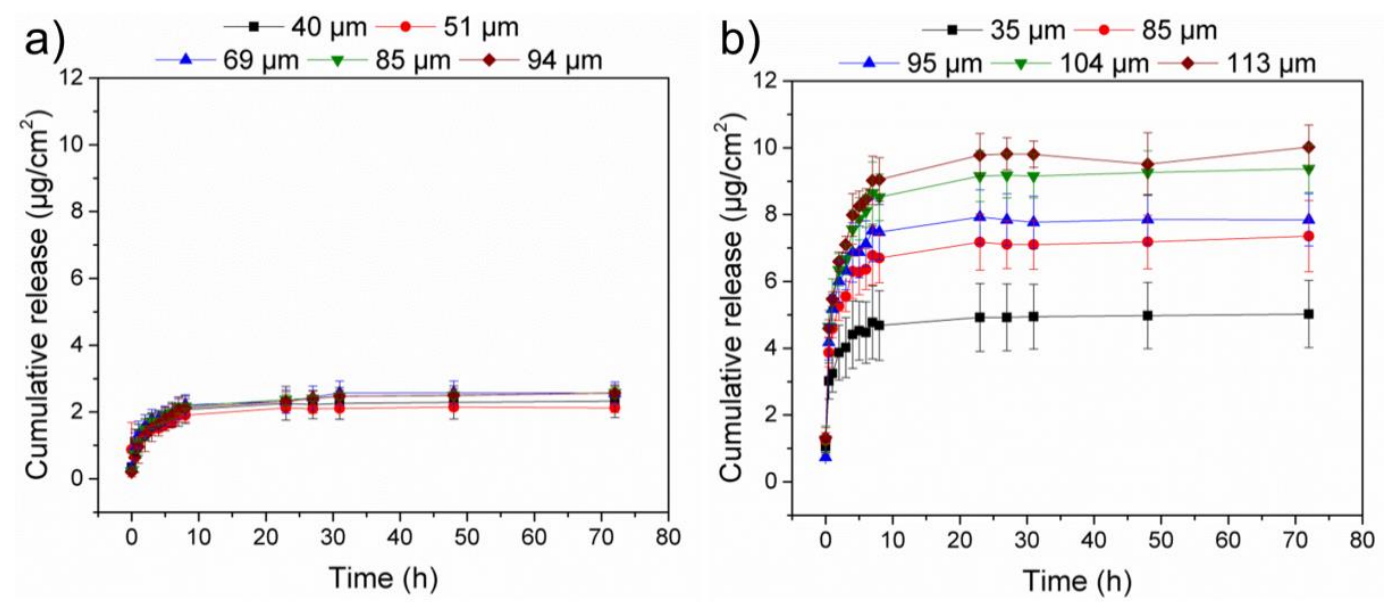

c)
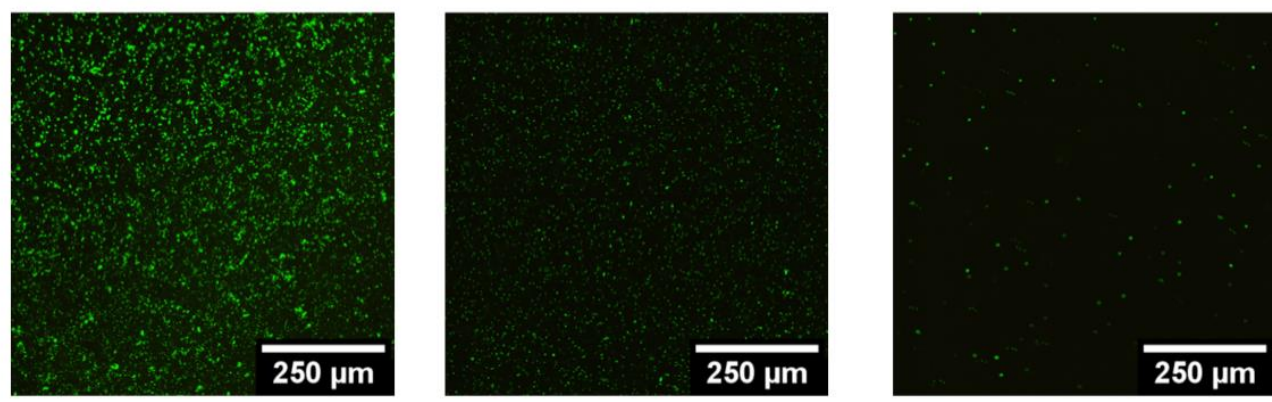

Control

Etched

Etched + Loaded

d)

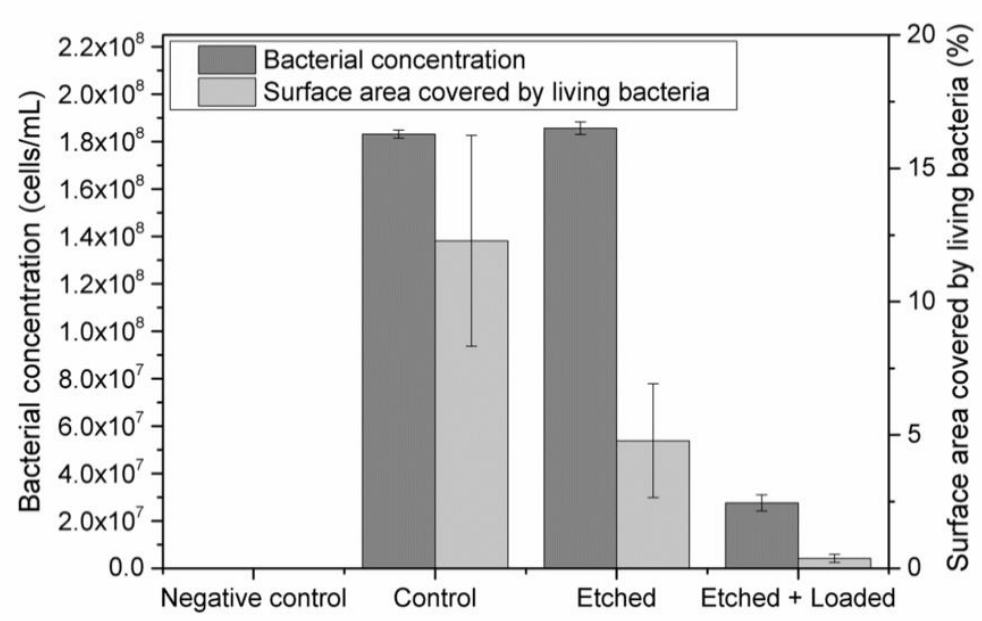

Figure 7. Cumulative drug release profiles from selectively etched zirconia toughened alumina samples loaded with a) gentamicin solution and b) gentamicin-encapsulated liposomes. Legend indicates the average thickness of the porous layer. Two samples had to be discarded because of large debinding defects. Results from E. coli cultures $(\mathrm{t}=4 \mathrm{~h})$ on the surface of "as sintered" (control), selectively etched, and selectively etched liposome-loaded zirconia toughened alumina samples (average porous layer thickness: $35 \mu \mathrm{m}$ ): c) fluorescence microscopy images of living bacteria on the surface; d) bacterial concentration in the medium monitored by flow cytometry and surface area covered by living bacteria. Error bars represent the standard deviations. 


\subsubsection{Biaxial flexural strength and ageing kinetics}

The thickness of the layer produced by selective etching was about $11 \mu \mathrm{m}$ for both the "as sintered+etched" and the "polished+annealed+etched" samples. Two-way ANOVA analysis of the strength testing results evidenced a significant main effect of polishing $(\mathrm{p}=0.007)$ and a significant main effect of selective etching ( $p=0.015)$, but no interaction $(p=0.307)$, which indicates that the effects of polishing and selective etching were independent. Etching induced a moderate decrease in the average strength, which was more important for the "polished+annealed" samples (-25\%) than for the "as sintered" samples (-11\%) (Table 6, Figure 9-a,b). This can be explained by the presence of pre-existing surface defects in the "as sintered" specimens: the impact of the new defects produced by etching is thus relatively less important in this case. Furthermore, for both the "as sintered" and "polished+annealed" surfaces, selective etching induced an increase in the Weibull modulus (Table 6) and no decrease in the minimum strength (Figure 9-b). Therefore, the flaws created by selective etching were not the most critical. Indeed, the "polished+annealed" group had the highest mean strength and $\sigma_{0}$ but the lowest Weibull modulus (Figure 9-a,b, Table 6), which suggests the presence of intrinsic defects in the bulk related to injection molding. The process would thus require further optimization to obtain samples with the same reliability as those obtained by conventional pressing.

Regarding phase transformation, it can be observed that selective etching induced a small increase in the initial monoclinic phase content in the "as sintered" samples ("as sintered": $V_{m}=$ 0.08; "as sintered+etched": $V_{m}=0.14$ ), but not in the "polished+annealed" samples, which contained a comparable amount of monoclinic phase ("polished+annealed": $V_{m}=0.18$; "polished+annealed+etched": $\left.V_{m}=0.16\right)$. In terms of kinetics, the trend was similar for all the 
groups tested and thus the ageing sensitivity does not appear to be significantly affected by selective etching (Figure 9-c).

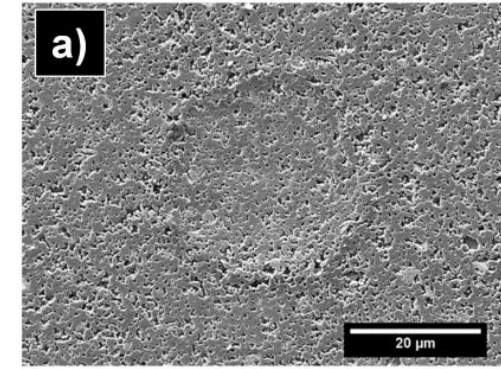

d)

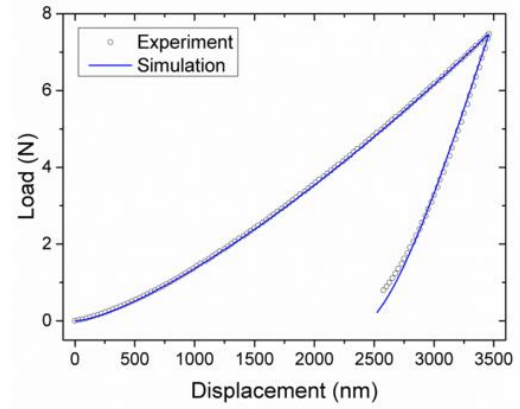

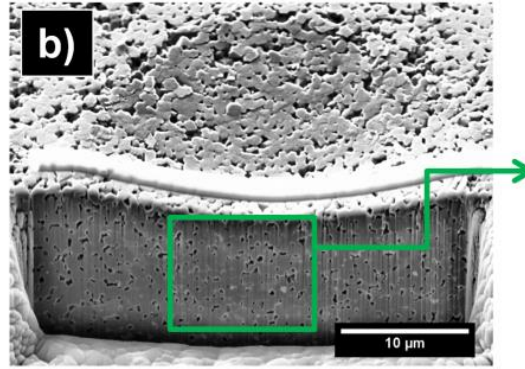

e)

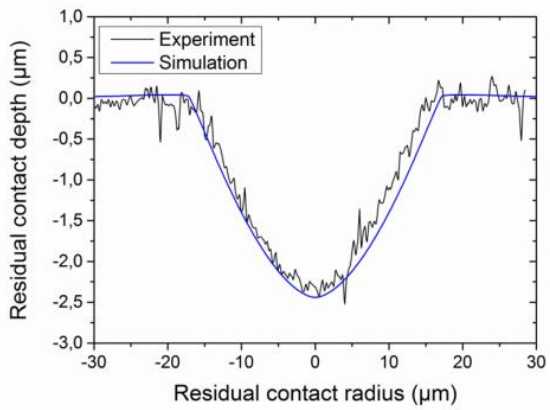

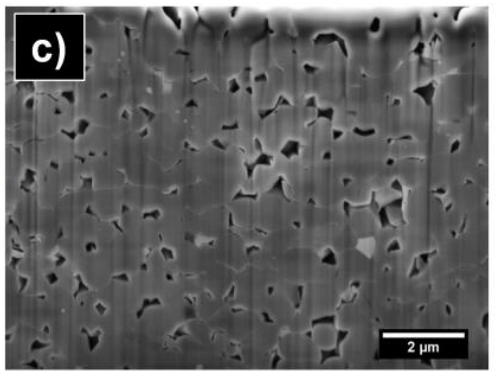

f)

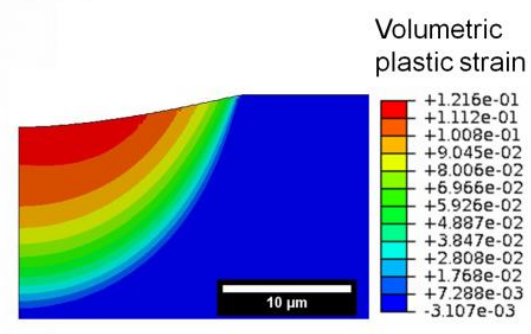

Figure 8. Results from spherical nanoindentation testing of a $27 \mu \mathrm{m}$ thick porous layer obtained by selective etching of polished zirconia toughened alumina: a) surface of an indentation observed by SEM; b), c) FIB cross-section of an indentation displaying densification of the porous layer which presents a quasi-plastic behavior; d) experimental (average) and simulated load-displacement curves; e) experimental (average) and simulated residual imprint; f) finite element calculation of the volumetric plastic strain (which corresponds to densification).

Table 5. Parameters identified by inverse finite element analysis describing the mechanical behavior of the porous layer obtained by selective etching

\begin{tabular}{|c|c|c|c|}
\hline Experimental curve & Lowest & Average & Highest \\
\hline$E(\mathrm{GPa})$ & 272 & 278 & 279 \\
\hline$\beta\left({ }^{\circ}\right)$ & 0.3 & 15.4 & 0.6 \\
\hline$\sigma_{\mathrm{c}}(\mathrm{MPa})$ & 3300 & 3210 & 3460 \\
\hline $\mathrm{p}_{\mathrm{b} 0}(\mathrm{MPa})$ & 4930 & 4680 & 5730 \\
\hline $\mathrm{D}\left(\mathrm{MPa}^{-1}\right)$ & $2.6 \times 10^{-4}$ & $5.3 \times 10^{-4}$ & $1.2 \times 10^{-3}$ \\
\hline
\end{tabular}


a)

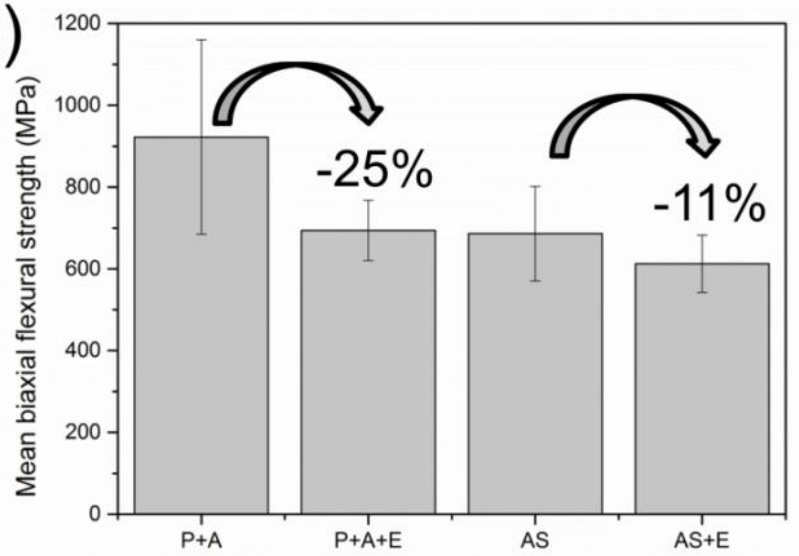

b)

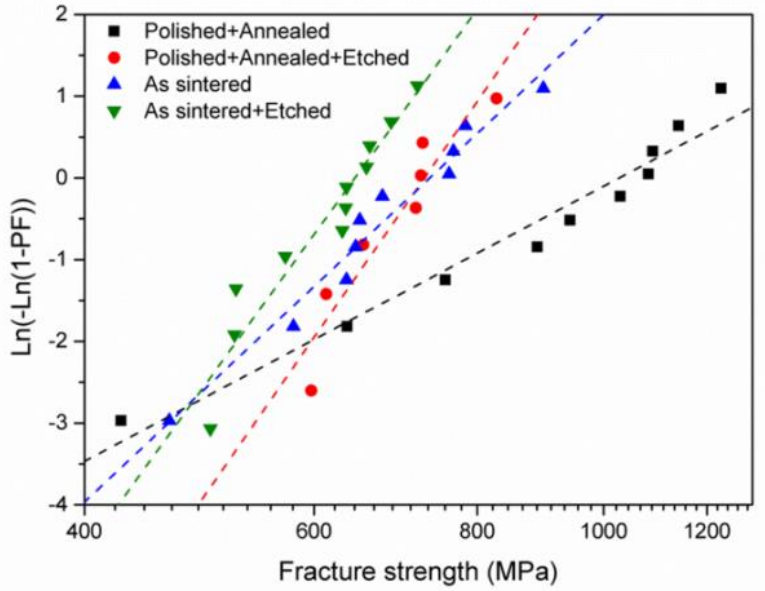

c)

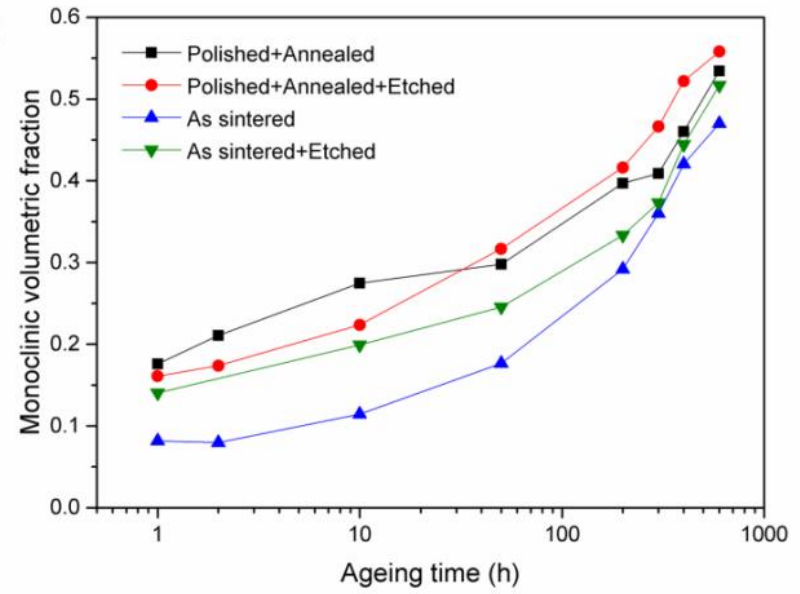

Figure 9. Assessment of the effect of selective etching on strength and ageing sensitivity of zirconia toughened alumina samples: a) mean strength (error bars represent the standard deviations), b) Weibull analysis of the strength, c) evolution of the monoclinic phase content during accelerated ageing tests. 
Table 6. Mean strength, Weibull modulus (biased) and Weibull characteristic strength resulting from 3-balls-on-3-balls testing of zirconia toughened alumina specimens

\begin{tabular}{|c|c|c|c|}
\hline Sample group & $\begin{array}{c}\sigma_{\text {mean }}[\mathrm{MPa}] \pm \\
\text { standard deviation }\end{array}$ & $\begin{array}{c}\mathrm{m}(90 \% \text { confidence } \\
\text { interval })\end{array}$ & $\begin{array}{c}\sigma_{0}[\mathrm{MPa}](90 \% \\
\text { confidence interval) }\end{array}$ \\
\hline Polished + Annealed & $922 \pm 238$ & $3.7(2-5)$ & $1029(865-1232)$ \\
\hline $\begin{array}{c}\text { Polished + Annealed } \\
\text { + Etched }\end{array}$ & $694 \pm 74$ & $10(4.6-14.1)$ & $729(671-796)$ \\
\hline As sintered & $686 \pm 115$ & $6.5(3.6-8.8)$ & $736(667-815)$ \\
\hline As sintered + Etched & $613 \pm 70$ & $9.5(5.5-12.8)$ & $669(628-714)$ \\
\hline
\end{tabular}

\section{Discussion}

The combination of surface modifications proposed in this work is promising for the design of ZTA components implantable in direct contact with bone, with tailored surface properties for promoting osseointegration and preventing infections.

Injection molding was shown to be a versatile process for surface micro-structuring, with a large possible range of values for roughness parameters (Figure 2). Additionally, as discussed in the introduction, it presents several advantages over other roughening treatments and has a high potential for industrialization. The values obtained for the average roughness $(\mathrm{Sa})$ might appear small as compared to the "moderately rough" range $(1 \mu \mathrm{m}-2 \mu \mathrm{m})$ that was suggested to induce the strongest bone response by Albrektsson and Wennerberg (2004) [67]. Nevertheless, the values reported in this study were numerically filtered, which is omitted by many authors. The $S a$ values obtained before filtering were substantially higher ("low" surface: $S_{a}=224 \pm 16 \mathrm{~nm}$; "medium" surface: $S_{a}=720 \pm 60 \mathrm{~nm}$; "high" surface: $S_{a}=1370 \pm 111 \mathrm{~nm}$ ), and it can thus be considered that the "high" surface falls within the recommended range. Finally, strength testing suggested the existence of intrinsic defects in the material related to injection molding (Figure 9- 
a,b). The presence of these defects shows the need for further optimization of the injection process to achieve mechanical performances similar to those obtained with standard processing (e.g., dry pressing).

On the other hand, selective etching is a totally novel process for ZTA, which can be used for two purposes:

- With a short etching time, it allows superposing of a substantial nano-roughness to the micro-topography produced by injection molding (Figure 3, Figure 4), without affecting the bulk of the material (Figure 6-a). According to the literature, this combination of micro- and nanoroughness should be favorable in terms of osseointegration [17-19]. Besides, the increase in the fluorine content of the surface (Figure 5) could enhance osteoblastic differentiation and interfacial bone formation as it does for titanium [68]. Finally, the selectively etched surface might reduce bacterial adhesion (Figure 7-c,d). This could be explained either by the nanoroughness (Figure 4) or the changes observed in the surface chemistry, in particular the increase in the fluorine content (Figure 5, Table 4), or a combination of both $[69,70]$.

- With a longer etching time, it allows the production of an interconnected nanoporous alumina layer with controlled thickness (Figure 6). As described previously, it has been suggested that nanoporous alumina can improve osteoblast adhesion and proliferation, increase matrix production and induce osteogenic differentiation [29-31]. Furthermore, the porous layer has the potential to be used as a carrier for drug delivery, providing antibacterial properties to the surface (Figure 7). The liposome encapsulation technique proposed in this work improves the loading of gentamicin. Besides, since liposomes can be loaded with both hydrophobic and hydrophilic molecules the method is potentially applicable to many other therapeutic agents [71] 
(for an example with vancomycin, see supplementary information). Nevertheless, the quantities loaded which represent about $0.5 \%-1 \%$ of the total porous volume available still appear small and there is thus room for optimization: in particular it should be possible to increase the quantity of drug loaded and the sustainability of the release by tuning liposome size.

The main benefit of selective etching when compared to other existing processes to produce nanoporous alumina on implants, such as for instance anodization [28,72], is that the layer obtained is not a coating. There is no interface with the bulk, which is highly beneficial in terms of reliability since it avoids any problem related to lack of adhesion or delamination. Furthermore, the contact behavior of the porous layer obtained is quasi-plastic (no cracks were observed under indentations). The yield stress $\left(\sigma_{y} \approx 3300 \mathrm{MPa}\right)$ and the indentation hardness (plateau at $8 \mathrm{GPa})$ are maintained at high values whereas the decrease in the elastic modulus $(E \approx$ $275 \mathrm{GPa}$ ) could be beneficial since it reduces the mismatch with the bone modulus (Figure 8, Table 5). Besides, it was shown that the presence of the porous layer does not impair flexural strength: the decrease in average strength, which was moderate, was compensated by an increase in Weibull modulus and the minimum strength was not affected (Figure 9, Table 6). Therefore, in a component larger than the tested specimens (e.g., an acetabular cup), the failure would likely be governed by intrinsic flaws, whose maximum size statistically increases with material volume. Since the pore size is too small for the cells to enter, the layer thickness tested $(11 \mu \mathrm{m})$ can be considered as a conservative estimate of the thickness necessary to promote osseointegration. Nevertheless, depending on the quantity of antibiotic which needs to be released and on the loading efficiency, a thicker layer might be required for drug loading. In this case, further study of the influence of the layer thickness on strength would be required. Finally, regarding phase transformation, the influence of selective etching is limited to a small increase in 
the initial volume of monoclinic phase content, without any impact on LTD kinetics. The excellent resistance to ageing of ZTA is thus not compromised by the treatment.

\section{Conclusion}

The combination of injection molding and selective etching allows the manufacture of ZTA samples with a substantial nano-roughness superposed to a controlled micro-topography. Selective etching also enables the formation of an interconnected porous alumina layer, which can be used as a carrier for drug delivery. The impact of selective etching on mechanical properties and hydrothermal stability is limited. Future studies will aim to optimize drug loading and to assess the impact of these surface modifications on cell and bone response. 


\section{Acknowledgement}

The authors would like to acknowledge the European Commission funding under the 7th Framework Programme (Marie Curie Initial Training Networks; grant number: 289958, Bioceramics for bone repair), the support of the Ministry of Economy and Competitiveness (MINECO) of Spain (project ref. MAT2014-60720-R) and the Government of Catalonia for the grant 2014SGR0137. The authors would also like to thank Dr. Trifon Trifonov for his help related to FIB sessions, Dr. Montserrat Dominguez for her help related to XPS analysis, and Dr. Yassine Maazouz and Dr. David Pastorino for their valuable advice regarding the drug delivery experiments.

\section{Disclosures}

Meinhard Kuntz is employed full time by the German company CeramTec GmbH, which produces ceramic components for joint replacements. Katia Biotteau was employed by CeramTec GmbH for 12 months. Other authors have no conflict of interest to declare.

\section{Abbreviations}

AFM, Atomic Force Microscopy; DPPC, 1,2-dipalmitoyl-sn-glycero-3-phosphocholine; FE, Finite Elements; FIB, Focused Ion Beam; HCl, Hydrochloric acid; HF, Hydrofluoric acid; LTD, Low Temperature Degradation; OPA, o-phtaldialdehyde; PBS, Phosphate Buffered Saline; SEM, Scanning Electron Microscopy; WLI, White Light Interferometry; XPS, X-ray Photoelectron Spectroscopy; Y-TZP, Yttria-stabilized Tetragonal Zirconia Polycrystal; ZTA, Zirconia Toughened Alumina 


\section{References}

[1] S.M. Kurtz, S. Kocagöz, C. Arnholt, R. Huet, M. Ueno, W.L. Walter, Advances in zirconia toughened alumina biomaterials for total joint replacement, J. Mech. Behav. Biomed. Mater. 31 (2014) 107-116.

[2] O. Roualdes, M.E. Duclos, D. Gutknecht, L. Frappart, J. Chevalier, D.J. Hartmann, In vitro and in vivo evaluation of an alumina-zirconia composite for arthroplasty applications, Biomaterials. 31 (2010) 2043-2054.

[3] G. Pezzotti, K. Yamada, A.A. Porporati, M. Kuntz, K. Yamamoto, Fracture toughness analysis of advanced ceramic composite for hip prosthesis, J. Am. Ceram. Soc. 92 (2009) $1817-1822$.

[4] A.H. De Aza, J. Chevalier, G. Fantozzi, M. Schehl, R. Torrecillas, Crack growth resistance of alumina, zirconia and zirconia toughened alumina ceramics for joint prostheses, Biomaterials. 23 (2002) 937-945.

[5] C. Piconi, G. Maccauro, F. Muratori, Alumina Matrix Composites in Arthroplasty, Key Eng. Mater. 284-286 (2005) 979-982. doi:10.4028/www.scientific.net/KEM.284-286.979.

[6] K. Lee, S.B. Goodman, Current state and future of joint replacements in the hip and knee., Expert Rev. Med. Devices. 5 (2008) 383-93.

[7] L.L. Hench, Bioceramics: From Concept to Clinic, J. Am. Ceram. Soc. 74 (1991) 14871510 .

[8] M. Sundfeldt, L. V Carlsson, C.B. Johansson, P. Thomsen, C. Gretzer, Aseptic loosening, not only a question of wear: a review of different theories., Acta Orthop. 77 (2006) 17797.

[9] R. Burgkart, E. Steinhauser, M. Grassel, M. Kuntz, Direct to bone - possible ceramic solutions for monolithic hip implants, Biol. Symp. 11 (2006) 259-262.

[10] S. Kurtz, Prevalence of Primary and Revision Total Hip and Knee Arthroplasty in the United States From 1990 Through 2002, J. Bone Jt. Surg. 87 (2005) 1487. doi:10.2106/JBJS.D.02441.

[11] K.J. Bozic, S.M. Kurtz, E. Lau, K. Ong, T.P. Vail, D.J. Berry, The Epidemiology of Revision Total Hip Arthroplasty in the United States, J. Bone Jt. Surg. 91 (2009) 128-133.

[12] K.J. Bozic, S.M. Kurtz, E. Lau, K. Ong, V. Chiu, T.P. Vail, H.E. Rubash, D.J. Berry, The epidemiology of revision total knee arthroplasty in the united states, in: Clin. Orthop. Relat. Res., 2010: pp. 45-51. 
[13] J. Raphel, M. Holodniy, S.B. Goodman, S.C. Heilshorn, Multifunctional Coatings to Simultaneously Promote Osseointegration and Prevent Infection of Orthopaedic Implants, Elsevier Ltd, 2016. doi:10.1016/j.biomaterials.2016.01.016.

[14] J.W. Costerton, Bacterial bofilms: a common cause of persistent infections, Science (80-. ). 284 (1999) 1318-1322.

[15] A.G. Gristina, P. Naylor, Q. Myrvik, Infections from biomaterials and implants: a race for the surface., Med. Prog. Technol. 14 (1987) 205-224.

[16] H.J. Busscher, H.C. van der Mei, G. Subbiahdoss, P.C. Jutte, J.J. a. M. van den Dungen, S. a. J. Zaat, M.J. Schultz, D.W. Grainger, Biomaterial-Associated Infection: Locating the Finish Line in the Race for the Surface, Sci. Transl. Med. 4 (2012) 153rv10-153rv10.

[17] C. Zink, H. Hall, D.M. Brunette, N.D. Spencer, Orthogonal nanometer-micrometer roughness gradients probe morphological influences on cell behavior., Biomaterials. 33 (2012) 8055-61.

[18] A. Wennerberg, T. Albrektsson, On implant surfaces: a review of current knowledge and opinions., Int. J. Oral Maxillofac. Implants. 25 (2009) 63-74.

[19] P.G. Coelho, R. Jimbo, N. Tovar, E. a Bonfante, Osseointegration: hierarchical designing encompassing the macrometer, micrometer, and nanometer length scales., Dent. Mater. 31 (2015) 37-52.

[20] Y.S. Park, S.H. Chung, W.J. Shon, Peri-implant bone formation and surface characteristics of rough surface zirconia implants manufactured by powder injection molding technique in rabbit tibiae, Clin. Oral Implants Res. 24 (2013) 586-591.

[21] R. Gadow, F. Kern, Pressureless Sintering of Injection Molded Zirconia Toughened Alumina Nanocomposites, J. Ceram. Soc. Japan. 114 (2006) 958-962.

[22] M.A. Elezz, F. Kern, R. Gadow, Manufacturing of ZTA composites for biomedical applications, in: Int. Conf. Eng. Technol. ICET 2012 - Conf. Bookl., 2012: pp. 10-14. doi:10.1109/ICEngTechnol.2012.6396123.

[23] F. Sommer, H. Walcher, F. Kern, M. Maetzig, R. Gadow, Influence of feedstock preparation on ceramic injection molding and microstructural features of zirconia toughened alumina, J. Eur. Ceram. Soc. 34 (2014) 745-751.

[24] S. Md Ani, A. Muchtar, N. Muhamad, J. a. Ghani, Fabrication of zirconia-toughened alumina parts by powder injection molding process: Optimized processing parameters, Ceram. Int. 40 (2014) 273-280. 
[25] S. Kapoor, R. Hegde, A.J. Bhattacharyya, Influence of surface chemistry of mesoporous alumina with wide pore distribution on controlled drug release, J. Control. Release. 140 (2009) 34-39.

[26] E. Gultepe, D. Nagesha, S. Sridhar, M. Amiji, Nanoporous inorganic membranes or coatings for sustained drug delivery in implantable devices, Adv. Drug Deliv. Rev. 62 (2010) 305-315.

[27] A. Krajewski, A. Ravaglioli, E. Roncari, P. Pinasco, L. Montanari, Porous ceramic bodies for drug delivery, J. Mater. Sci. Mater. Med. 11 (2000) 763-771.

[28] A.R. Walpole, Z. Xia, C.W. Wilson, J.T. Triffitt, P.R. Wilshaw, A novel nano-porous alumina biomaterial with potential for loading with bioactive materials, J. Biomed. Mater. Res. - Part A. 90 (2009) 46-54.

[29] S. Pujari-Palmer, T. Lind, W. Xia, L. Tang, M.K. Ott, Controlling Osteogenic Differentiation through Nanoporous Alumina, J. Biomater. Nanobiotechnol. 5 (2014) 98104. doi:10.4236/jbnb.2014.52012.

[30] K.C. Popat, E.E. Leary, V. Mukhatyar, K. Chatvanichkul, G.K. Mor, C.A. Grimes, T.A. Desai, Influence of nanoporous alumina membranes on long-term osteoblast response, 26 (2005) 4516-4522. doi:10.1016/j.biomaterials.2004.11.026.

[31] K. Popat, Osteogenic differentiation of marrow stromal cells cultured on nanoporous alumina, J. Biomed. Mater. Res. A. 81 (2007) 771-780.

[32] S. Usami, H. Kimoto, I. Takahashi, S. Shida, Strength of ceramic materials containing small flaws, Eng. Fract. Mech. 23 (1986) 745-761.

[33] E. Ryshkewitch, Compression Strength of Porous Sintered Alumina and Zirconia, J. Am. Ceram. Soc. 36 (1953) 65-68.

[34] B.A. Latella, B.H. OConnor, N.P. Padture, B.R. Lawn, Hertzian Contact Damage in Porous Alumina Ceramics, J. Am. Ceram. Soc. 80 (2005) 1027-1031. doi:10.1111/j.11512916.1997.tb02940.x.

[35] A.P. Roberts, E.J. Garboczi, Elastic Properties of Model Porous Ceramics, J. Am. Ceram. Soc. 83 (2000) 3041-3048.

[36] A.H. Heuer, Transformation Toughening in ZrO2-Containing Ceramics, J. Am. Ceram. Soc. 70 (1987) 689-698.

[37] S. Lawson, Environmental degradation of zirconia ceramics, J. Eur. Ceram. Soc. 15 (1995) 485-502.

[38] J. Chevalier, What future for zirconia as a biomaterial?, Biomaterials. 27 (2006) 535-543. 
[39] P. Fabbri, C. Piconi, E. Burresi, G. Magnani, F. Mazzanti, C. Mingazzini, Lifetime estimation of a zirconia-alumina composite for biomedical applications, Dent. Mater. 30 (2014) 138-142.

[40] S. Deville, J. Chevalier, G. Fantozzi, J.F. Bartolomé, J. Requena, J.S. Moya, R. Torrecillas, L.A. Díaz, Low-temperature ageing of zirconia-toughened alumina ceramics and its implication in biomedical implants, J. Eur. Ceram. Soc. 23 (2003) 2975-2982.

[41] J. Chevalier, S. Grandjean, M. Kuntz, G. Pezzotti, On the kinetics and impact of tetragonal to monoclinic transformation in an alumina/zirconia composite for arthroplasty applications, Biomaterials. 30 (2009) 5279-5282.

[42] V. Lowalekar, S. Raghavan, Etching of Zirconium Oxide, Hafnium Oxide, and Hafnium Silicates in Dilute Hydrofluoric Acid Solutions, J. Mater. Res. 19 (2004) 1149-1156.

[43] K.R. Williams, K. Gupta, M. Wasilik, Etch rates for micromachining processing - Part II, J. Microelectromechanical Syst. 12 (2003) 761-778.

[44] A. Wennerberg, T. Albrektsson, A.T. Wennerberg A, Suggested guidelines for the topographic evaluation of implant surfaces., Int. J. Oral Maxillofac. Implants. 15 (2000) 331-344.

[45] L.M. Svanborg, M. Andersson, A. Wennerberg, Surface characterization of commercial oral implants on the nanometer level, J. Biomed. Mater. Res. - Part B Appl. Biomater. 92 (2010) 462-469.

[46] W.P. Dong, P.J. Sullivan, K.J. Stout, Comprehensive study of parameters for characterising three- dimensional surface topography. III: Parameters for characterising amplitude and some functional properties, Wear. 178 (1994) 29-43.

[47] W.P. Dong, P.J. Sullivan, K.J. Stout, Comprehensive study of parameters for characterising three-dimensional surface topography. IV: Parameters for characterising spatial and hybrid properties, Wear. 178 (1994) 45-60.

[48] K.J. Stout, L. Blunt, Three Dimensional Surface Topography, Elsevier, 2000. doi:10.1016/B978-185718026-8/50119-3.

[49] S.S. Sampath, D.H. Robinson, Comparison of new and existing spectrophotometric methods for the analysis of tobramycin and other aminoglycosides, J. Pharm. Sci. 79 (1990) 428-431.

[50] P. Frutos Cabanillas, E. Díez Peña, J.M. Barrales-Rienda, G. Frutos, Validation and in vitro characterization of antibiotic-loaded bone cement release, Int. J. Pharm. 209 (2000) 15-26. 
[51] J. Gubernator, Z. Drulis-Kawa, A. Kozubek, A simply and sensitive fluorometric method for determination of gentamicin in liposomal suspensions, Int. J. Pharm. 327 (2006) 104109.

[52] B. Zmistowski, C.J. Fedorka, E. Sheehan, G. Deirmengian, M.S. Austin, J. Parvizi, Prosthetic joint infection caused by gram-negative organisms., J. Arthroplasty. 26 (2011) 104-108.

[53] T.N. Peel, A.C. Cheng, K.L. Buising, P.F.M. Choong, Microbiological aetiology, epidemiology, and clinical profile of prosthetic joint infections: Are current antibiotic prophylaxis guidelines effective?, Antimicrob. Agents Chemother. 56 (2012) 2386-2391.

[54] D. Rodríguez-Pardo, C. Pigrau, J. Lora-Tamayo, A. Soriano, M.D. del Toro, J. Cobo, J. Palomino, G. Euba, M. Riera, M. Sánchez-Somolinos, N. Benito, M. FernándezSampedro, L. Sorli, L. Guio, J.A. Iribarren, J.M. Baraia-Etxaburu, A. Ramos, A. Bahamonde, X. Flores-Sánchez, P.S. Corona, J. Ariza, C. Amat, M.N. Larrosa, M. Puig, O. Murillo, X. Cabo, M.Á. Goenaga, M. Elola, G. De la Herrán, J.M. Garcia-Arenzana, S. García-Ramiro, J.C. Martínez-Pastor, E. Tornero, J.M. García-Lechuz, M. Marín, M. Villanueva, I. López, R. Cisterna, J.M. Santamaría, M.J. Gómez, A. Puente, P. Cano, J.P. Horcajada, P. González-Mínguez, E. Portillo, L. Puig, M. Franco, M. Jordán, P. Coll, J. Amador-Mellado, C. Fuster-Foz, L. García-Paíno, I. Nieto, M.Á. Muniain, A.I. Suárez, J. Praena, M.J. Gómez, A. Puente, M.A. Maseguer, E. Garagorri, V. Pintado, C. Marinescu, A. Ramírez, F. Montaner, E. Múñez, T. Álvarez, R. García, E. Puente, C. Salas, M.C. Fariñas, J.M. Pérez, B.V. Achabal, J.M. Montejo Baranda, Gram-negative prosthetic joint infection: Outcome of a debridement, antibiotics and implant retention approach. A large multicentre study, Clin. Microbiol. Infect. 20 (2014) O911-O919.

[55] A.J. Moseson, S. Basu, M.W. Barsoum, Determination of the effective zero point of contact for spherical nanoindentation, J. Mater. Res. 23 (2008) 204-209.

[56] L.H. He, N. Fujisawa, M. V Swain, Elastic modulus and stress-strain response of human enamel by nano-indentation, Biomaterials. 27 (2006) 4388-4398.

[57] P. Clément, S. Meille, J. Chevalier, C. Olagnon, Mechanical characterization of highly porous inorganic solids materials by instrumented micro-indentation, Acta Mater. 61 (2013) 6649-6660.

[58] D. Staub, S. Meille, V. Le Corre, L. Rouleau, J. Chevalier, Identification of a damage criterion of a highly porous alumina ceramic, Acta Mater. 107 (2016) 261-272.

[59] F.L. DiMaggio, I.S. Sandler, Material model for granular soils, J. Eng. Mech. Div. 97 (1971) 935-950.

[60] J. Chevalier, C. Olagnon, G. Fantozzi, Study of the residual stress field around Vickers indentations in a 3Y-TZP, J. Mater. Sci. 31 (1996) 2711-2717. 
[61] T. Fett, G. Rizzi, E. Ernst, R. M??ller, R. Oberacker, A 3-balls-on-3-balls strength test for ceramic disks, J. Eur. Ceram. Soc. 27 (2007) 1-12.

[62] T. Fett, G. Rizzi, 3-balls-on-3-balls test for ceramic disks: A finite element study, 2004.

[63] D. Munz, T. Fett, Ceramics: mechanical properties, failure behaviour, materials selection, Springer Science \& Business Media, 1999.

[64] H. Toraya, M. Yoshimura, S. Somiya, Calibration Curve for Quantitative Analysis of the Monoclinic-Tetragonal ZrO2 System by X-Ray Diffraction, Commun. Am. Ceram. Soc. 67 (1984) 119-121.

[65] Q. Flamant, F. García Marro, J.J. Roa Rovira, M. Anglada, Hydrofluoric acid etching of dental zirconia. Part 1: Etching mechanism and surface characterization, J. Eur. Ceram. Soc. 36 (2016) 121-134.

[66] D. Majumdar, D. Chatterjee, X-ray photoelectron spectroscopic studies on yttria, zirconia, and yttria-stabilized zirconia, J. Appl. Phys. 70 (1991) 988-992. doi:10.1063/1.349611.

[67] T. Albrektsson, A. Wennerberg, Oral implant surfaces: Part 1--review focusing on topographic and chemical properties of different surfaces and in vivo responses to them., Int. J. Prosthodont. 17 (2004) 536-43.

[68] L. Cooper, Y. Zhou, J. Takebe, J. Guo, A. Abron, A. Holmen, J. Ellingsen, Fluoride modification effects on osteoblast behavior and bone formation at $\mathrm{TiO}$ grit-blasted c.p. titanium endosseous implants, Biomaterials. 27 (2006) 926-936.

[69] K. Bazaka, R.J. Crawford, E.P. Ivanova, Do bacteria differentiate between degrees of nanoscale surface roughness?, Biotechnol. J. 6 (2011) 1103-1114.

[70] M. Yoshinari, Y. Oda, T. Kato, K. Okuda, Influence of surface modifications to titanium on antibacterial activity in vitro, Biomaterials. 22 (2001) 2043-2048.

[71] H. Pinto-Alphandary, A. Andremont, P. Couvreur, Targeted delivery of antibiotics using liposomes and nanoparticles: Research and applications, Int. J. Antimicrob. Agents. 13 (2000) 155-168.

[72] A.R. Walpole, E.P. Briggs, M. Karlsson, E. Pålsgård, P.R. Wilshaw, Nano-porous Alumina Coatings for Improved Bone Implant Interfaces, in: Materwiss. Werksttech., 2003: pp. 1064-1068.

[73] S. Dolarevic, A. Ibrahimbegovic, A modified three-surface elasto-plastic cap model and its numerical implementation, Comput. Struct. 85 (2007) 419-430. 


\section{Appendix A - Modified Drucker-Prager/cap model [58]}

The Drucker-Prager failure surface is defined in the meridional $(p-t)$ plane by:

$$
F_{s}=t-p \tan \beta-d=0
$$

where $p$ is the equivalent pressure stress, $t$ is the deviatoric stress, $\beta$ is the angle of friction of the material and $d$ is its cohesion which can be expressed as a function of the yield stress in simple compression $\sigma_{c}$ as:

$$
d=\left(1-\frac{1}{3} \tan \beta\right) \sigma_{c}
$$

The cap yield failure surface, which binds the yield surface in hydrostatic compression, is defined in the p-t plane by:

$$
F_{c}=\sqrt{\left(p-p_{a}\right)^{2}+\left(\frac{R t}{1+\alpha-\frac{\alpha}{\cos \beta}}\right)^{2}}-R\left(d+p_{a} \tan \beta\right)=0
$$

where $R$ is the cap eccentricity (which controls the shape of the cap), $\alpha$ is a small number (typically comprised between 0.01 and 0.05 ) used to define the transition yield surface and $p_{a}$ is an evolution parameter that represents the volumetric inelastic strain driven hardening given as:

$$
p_{a}=\frac{p_{b}-R d}{(1+R \tan \beta)}
$$

where $p_{b}$ is the hydrostatic compression yield stress. In this work, the evolution of $p_{b}$ was modeled with the following law:

$$
p_{b}\left(\varepsilon_{V}\right)=\frac{1}{D} \ln \left(1+\frac{\varepsilon_{V}}{W}\right)+P_{b 0}
$$


where $\varepsilon_{V}$ is the hydrostatic plastic strain, $p_{b 0}$ the initial hydrostatic compression yield stress, $W$ is the maximum plastic volumetric strain (which theoretically corresponds to the porosity) and $D$ is the maximum plastic volumetric strain rate [73].

To provide a smooth intersection between the cap and the Drucker-Prager failure surfaces, a transition yield surface is defined as:

$$
F_{t}=\sqrt{\left(p-p_{a}\right)^{2}+\left[t-\left(1-\frac{\alpha}{\cos \beta}\right)\left(d+p_{a} \tan \beta\right)\right]^{2}}-\alpha\left(d+p_{a} \tan \beta\right)=0
$$

The plastic flow is defined by a flow potential that is associated in the cap region in the meridional plane and non-associated in the Drucker-Prager failure surface and transition regions. It consists in an elliptical portion in the cap region that is identical to the cap yield surface:

$$
G_{c}=\sqrt{\left(p-p_{a}\right)^{2}+\left(\frac{R t}{1+\alpha-\frac{\alpha}{\cos \beta}}\right)^{2}}
$$

and another elliptical portion in the failure and transition regions:

$$
G_{s}=\sqrt{\left[\left(p_{a}-p\right) \tan \beta\right]^{2}+\left(\frac{t}{1+\alpha-\frac{\alpha}{\cos \beta}}\right)^{2}}
$$

